\title{
SURFACE ENERGIES IN A TWO-DIMENSIONAL MASS-SPRING MODEL FOR CRYSTALS
}

\author{
FLORIAN THEIL ${ }^{1}$
}

\begin{abstract}
We study an atomistic pair potential-energy $E^{(n)}(y)$ that describes the elastic behavior of two-dimensional crystals with $n$ atoms where $y \in \mathbb{R}^{2 \times n}$ characterizes the particle positions. The main focus is the asymptotic analysis of the ground state energy as $n$ tends to infinity. We show in a suitable scaling regime where the energy is essentially quadratic that the energy minimum of $E^{(n)}$ admits an asymptotic expansion involving fractional powers of $n$ :

$$
\min _{y} E^{(n)}(y)=n E_{\mathrm{bulk}}+\sqrt{n} E_{\text {surface }}+o(\sqrt{n}), \quad n \rightarrow \infty .
$$

The bulk energy density $E_{\mathrm{bulk}}$ is given by an explicit expression involving the interaction potentials. The surface energy $E_{\text {surface }}$ can be expressed as a surface integral where the integrand depends only on the surface normal and the interaction potentials. The evaluation of the integrand involves solving a discrete algebraic Riccati equation. Numerical simulations suggest that the integrand is a continuous, but nowhere differentiable function of the surface normal.
\end{abstract}

\section{Mathematics Subject Classification. 74Q05.}

Received January 29, 2010. Revised October 28, 2010.

Published online February 23, 2011.

\section{INTRODUCTION}

\subsection{Previous work and concepts}

The objective of this paper is to provide mathematical analysis of surface energy densities associated to the ground state energies of atomistic systems in the thermodynamic limit where the number of particles becomes large.

To be more precise, we consider an atomistic pair-interaction model where for a finite lattice $\mathcal{L} \subset \mathbb{Z}^{d}$ the energy of a lattice deformation $y: \mathbb{Z}^{d} \rightarrow \mathbb{R}^{d}$ can be written as a sum of pair-interactions

$$
E^{(\mathcal{L})}(\{y\}):=\frac{1}{2} \sum_{x, x^{\prime} \in \mathcal{L}} V_{\left|x-x^{\prime}\right|}\left(\left|y(x)-y\left(x^{\prime}\right)\right|\right),
$$

Keywords and phrases. Continuum mechanics, difference equations.

1 Mathematics Institute, University of Warwick, Coventry, CV4 7AL, UK. f.theil@warwick.ac.uk 
the factor $\frac{1}{2}$ ensures that each bond is counted only once. The potential $V_{\left|x-x^{\prime}\right|}\left(\left|y(x)-y\left(x^{\prime}\right)\right|\right)$ is the interaction energy of the particle pair $x, x^{\prime} \in \mathbb{Z}^{d}$ and may depend on the particle labels $x, x^{\prime}$. This energy is a realistic model for a crystal in the sense that it is invariant under rotations and translations, i.e. $E^{(\mathcal{L})}(\{y\})=E^{(\mathcal{L})}(\{R y+t\})$ for all $R \in S O(d)$ and $t \in \mathbb{R}^{d}$.

The properties of such systems has been studied extensively, e.g. in $[1,12,19,20]$. At leading order the behavior of the energy $E^{(\mathcal{L})}$ is dominated by the bulk energy density, i.e. the limiting energy per particle as the the size of $\mathcal{L}$ tends to infinity, e.g. $[3,12]$.

The first correction which accounts for the finiteness of the number of particles are caused by the presence of surfaces. Explicit expressions for surface energies are important in many physical, biological and mathematical applications. Examples are nanoclusters, biological membranes and image reconstruction. They have been derived previously for various, mostly one-dimensional models, $c f$. $[3,6,9,18]$. Depending on the details of the model, surfaces can be dominated by one of several effects

The energy increases due to "missing" bonds. In [3] this effect is carefully analyzed under the additional assumption that all the particles are clamped according to a continuum map $\phi$. Within such a setting it is possible to define bulk and surface energies in a very simple way. We give a slightly simplified account of their results.

Definition 1.1. For $\nu \in \mathbb{Z}^{d} \backslash\{0\}$ and $F \in \mathbb{R}^{d \times d}$ the clamped bulk and surface energy density are given by

$$
\begin{aligned}
W_{\mathrm{cl}}(F) & :=\frac{1}{2} \sum_{x \in \mathbb{Z}^{d}} V_{|x|}(|F x|), \\
W_{\mathrm{cl}}^{s}(F, \nu) & :=-\lim _{\rho \rightarrow \infty} \frac{1}{2 \rho} \sum_{\substack{x, x^{\prime} \in \mathbb{Z}^{d} \cap B(0, \rho) \\
x \cdot \nu \leq 0, x^{\prime} \cdot \nu>0}} V_{\left|x-x^{\prime}\right|}\left(\left|F\left(x-x^{\prime}\right)\right|\right) .
\end{aligned}
$$

Both energy densities rely on the assumption that particle positions are clamped to an affinely deformed lattice. The result by Blanc, Le Bris and Lions demonstrates that those energies contain enough information to characterize the energy defect of deformations which are clamped according to a continuum map. The version below is slightly weaker than the actual result.

Theorem 1.2. Let $\Omega \subset \mathbb{R}^{d}$ be a domain such that $\partial \Omega$ is a polygon with rational normals, $\mathcal{L}^{(\rho)}=\rho \Omega \cap \mathbb{Z}^{d}$ (cf. Fig. 1) and $\phi \in C^{\infty}\left(\Omega, \mathbb{R}^{d}\right)$ a continuum deformation. Then

$$
\begin{aligned}
E^{(\rho)}\left(\left\{\rho \phi\left(\frac{1}{\rho} \cdot\right)\right\}\right) & =\frac{1}{2} \sum_{x, x^{\prime} \in \mathcal{L}(\rho)} V_{\left|x-x^{\prime}\right|}\left(\rho\left|\phi\left(\frac{1}{\rho} x\right)-\phi\left(\frac{1}{\rho} x^{\prime}\right)\right|\right) \\
& =\frac{\# \mathcal{L}^{(\rho)}}{|\Omega|} \int_{\Omega} W_{\mathrm{cl}}(\nabla \phi) \mathrm{d} x+\rho^{d-1} \int_{\partial \Omega} W_{\mathrm{cl}}^{s}(\nabla \phi(s), \nu(s)) \mathrm{d} \mathcal{H}^{d-1}(s)+o\left(\rho^{d-1}\right),
\end{aligned}
$$

as $\rho \rightarrow \infty$, where $\nu(x) \in \mathbb{Z}^{2} \backslash\{0\}$ is a surface normal at $x \in \partial \Omega$.

Under less restrictive assumptions on the configuration of the particles the energy defect is strictly smaller than $W_{\mathrm{cl}}^{s}$. This is caused by the fact that energy is gained by if the particles relax to nontrivial positions near the surface. In general this could lead to changes of the lattice structure. To limit the complexity of the analysis we restrict ourselves to the case where only small strains are admissible, and as a consequence the nearest-neighbor structure is unchanged. In the context of one-dimensional models this effect has received significant attention in the literature, e.g. [4,9].

In higher dimensional situations surface energies have striking implications on the macroscopic properties of the continuum limit. Examples are the early growth stages of crystal which can are determined by the Wulff construction, see e.g. [11] and the references therein. Important results in this direction have been obtained by several authors, $c f .[1,20]$. 


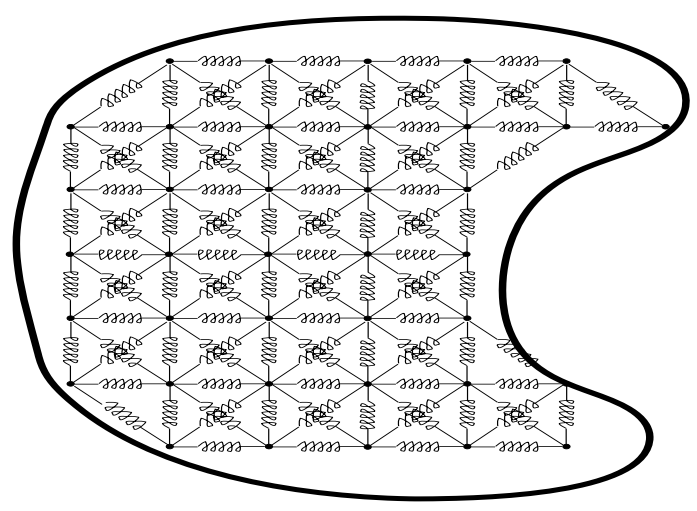

Figure 1. The discrete domain $\mathcal{L}^{(\rho)}$.

Here, we provide mathematical analysis of the surface energy in the case of two-dimensional atomistic energy which is invariant under translations and rotations. We will demonstrate that the surface energy is local, i.e. it can be written in the form of a surface integral. The integrand (surface energy density) is determined by a system of algebraic equation, the discrete Riccati equation. Solving such equations is a standard problem in optimal control theory and we can use numerical approximations to discuss the dependency of the surface energy on the surface normal.

\subsection{Description of the model and main result}

We restrict our attention to the two-dimensional setting where the surface energy turns into a perimeter energy.

The main motivation for this assumption is that it simplifies the notation on several occasions.

We assume that only those maps $y: \mathbb{Z}^{2} \rightarrow \mathbb{R}^{2}$ compete in the energy minimization which have the property that for each triple $\left\{x_{1}, x_{2}, x_{3}\right\} \subset \mathbb{Z}^{2}$ the oriented area of the triangle with the corners $y\left(x_{1}\right), y\left(x_{2}\right), y\left(x_{3}\right)$ is nonnegative

$$
\begin{aligned}
\mathcal{A}:= & \left\{y: \mathbb{Z}^{2} \rightarrow \mathbb{R}^{2} \mid \operatorname{det}\left(y\left(x_{2}\right)-y\left(x_{1}\right), y\left(x_{3}\right)-y\left(x_{1}\right)\right) \geq 0 \text { for all }\left\{x_{1}, x_{2}, x_{3}\right\} \subset \mathbb{Z}^{2}\right. \\
& \text { such that } \left.\operatorname{diam}\left\{x_{1}, x_{2}, x_{3}\right\}=\sqrt{2} \text { and } \operatorname{det}\left(x_{2}-x_{1}, x_{3}-x_{1}\right) \geq 0\right\} .
\end{aligned}
$$

Maps $y \in \mathcal{A}$ are called admissible. This assumption is natural as it rules out self-interpenetration. It plays a crucial role in Section 3 in connection with rigidity estimates.

In order to define a thermodynamic limit we fix a continuum domain $\Omega \subset \mathbb{R}^{2}$ and consider the family of finite lattices

$$
\mathcal{L}^{(\rho)}=\rho \Omega \cap \mathbb{Z}^{2}, \quad \rho \in(0, \infty)
$$

For an admissible lattice deformation $y \in \mathcal{A}$ we define the pair energy

$$
E^{(\rho)}(\{y\})=\sum_{x, x^{\prime} \in \mathcal{L}^{(\rho)}} V_{\left|x-x^{\prime}\right|}\left(\left|y(x)-y\left(x^{\prime}\right)\right|\right),
$$


where the potentials $V_{\lambda}(\cdot) \in C^{2}((0, \infty))$ satisfy

$$
\begin{aligned}
& V_{\lambda} \equiv 0 \text { if } \lambda \notin\{1, \sqrt{2}\}, \\
& V_{1}^{\prime}(1)+\sqrt{2} V_{\sqrt{2}}^{\prime}(\sqrt{2})=0, \\
& \min _{\lambda \in\{1, \sqrt{2}\}, r>0} V_{\lambda}^{\prime \prime}(r) \geq \gamma \text { for some constant } \gamma>0 .
\end{aligned}
$$

The condition involving the first derivatives of the potentials implies that the identity map $y(x)=x$ minimizes the energy per particle among all homogeneous dilatations $y(x)=r x$ in the limit $\rho \rightarrow \infty$ where $r \in(0, \infty)$ is an arbitrary dilation parameter.

The analysis in [12] is concerned with the ground states subject to affine boundary conditions. By the boundary $\partial \mathcal{L} \subset \mathcal{L} \subset \mathbb{Z}^{2}$ we mean those points $x \in \mathcal{L}$ that have an incomplete neighborhood in the sense that $\#(B(x, \sqrt{2}) \cap \mathcal{L})<9$, where $B(x, r)$ is the closed disk with radius $r$ centered at $x$. The strength of the internal stresses is measured by $\varepsilon=V_{1}^{\prime}(1)-\sqrt{2} V_{\sqrt{2}}^{\prime}(\sqrt{2})$. The key result of [12] is that there exists a constant $\varepsilon_{0}>0$ such that ground states subject to affine boundary conditions are periodic, provided that $|\varepsilon|<\varepsilon_{0}$. The result is sharp in the sense that it is possible to construct two convex quadratic potentials which satisfy (1.2)-(1.4) and non-affine deformations $y^{(\rho)} \in \mathcal{A}$ that satisfy the boundary condition $y^{(\rho)}(x)=x$ for $x \in \partial \mathcal{L}^{(\rho)}$ such that $\lim \sup _{\rho \rightarrow \infty} \frac{1}{\# \mathcal{L}^{(\rho)}} E^{(\rho)}\left(\left\{y^{(\rho)}\right\}\right)$ is strictly smaller than $W_{\mathrm{cl}}(\mathrm{Id})$.

Here, our main objective is to analyze the correction term $\min _{y \in \mathcal{A}} \frac{1}{\rho}\left(E^{(\rho)}(\{y\})-W_{\mathrm{cl}}(\mathrm{Id}) \# \mathcal{L}^{(\rho)}\right)$ in the limit as $\rho \rightarrow \infty$. We consider the case where only a finite number of normal vectors occurs and the internal stresses are small.

Definition 1.3. We say that an open set $\Omega \subset \mathbb{R}^{2}$ has a polygonal boundary with rational normals if $\partial \Omega=$ $\cup_{i=1}^{\# \text { edges }} \Gamma_{i}$, where $\mathcal{H}^{1}\left(\Gamma_{i} \cap \Gamma_{j}\right)=0$ if $i \neq j$ and there exist outward pointing normal vectors $\nu_{i} \in \mathbb{Z}^{2} \backslash\{0\}$, such that $\left(x_{i}-x_{i}^{\prime}\right) \cdot \nu_{i}=0$ for all $x_{i}, x_{i}^{\prime} \in \Gamma_{i}$.

Note that we do not follow the convention that $\nu$ has unit-length. The main result is that the surface energy can be written as a surface integral in the limit $\rho \rightarrow \infty$ provided that $\varepsilon=o(1)$ as $\rho \rightarrow \infty$.

Theorem 1.4 (main result). Let $K_{1}, K_{2}, \beta_{0} \in(0, \infty)$ be three real constants and assume that the pair potentials $V_{1}, V_{\sqrt{2}} \in C^{2}\left((0, \infty) \times\left(-\beta_{0}, \beta_{0}\right)\right)$ satisfy $(1.2)-(1.4)$, and

$$
\begin{aligned}
& V_{\lambda}^{\prime}(\lambda) \neq 0 \text { if } \beta \neq 0, \quad \lim _{\beta \rightarrow 0} V_{\lambda}^{\prime}(\lambda)=0, \\
& \lim _{\beta \rightarrow 0} V_{\lambda}^{\prime \prime}(\lambda)=K_{\lambda^{2}}, \quad \lambda \in\{1, \sqrt{2}\} .
\end{aligned}
$$

(The dependency of $V_{\lambda}$ on the second argument $\beta$ is not shown.) There exists a function $W_{\text {rel }}^{s}: \mathbb{Z}^{2} \backslash\{0\} \rightarrow \mathbb{R}$ which is defined later in (2.13) and can be interpreted as the energy of surface relaxation per unit length of perimeter with normal $\nu$, having the following property. For every open, bounded, simply connected domain $\Omega \subset \mathbb{R}^{2}$ such that the boundary $\partial \Omega$ is a polygon with rational normals and every sequence $\beta_{\rho}=o(1), \rho \rightarrow \infty$ the following equation holds

$$
\lim _{\rho \rightarrow \infty} \sigma_{\rho}=\int_{\partial \Omega} W_{\text {rel }}^{s}(\nu(x)) \mathrm{d} \mathcal{H}^{1}(x),
$$

where

$$
\sigma_{\rho}=\frac{1}{\varepsilon^{2} \rho}\left(\min _{y \in \mathcal{A}} E^{(\rho)}(\{y\})-W_{\mathrm{cl}}(\mathrm{Id}) \# \mathcal{L}^{(\rho)}-\rho \int_{\partial \Omega} W_{\mathrm{cl}}^{s}(\mathrm{Id}, \nu(s)) \mathrm{d} \mathcal{H}^{1}(s)\right)
$$

and

$$
\varepsilon=V_{1}^{\prime}(1)-\sqrt{2} V_{\sqrt{2}}^{\prime}(\sqrt{2})
$$




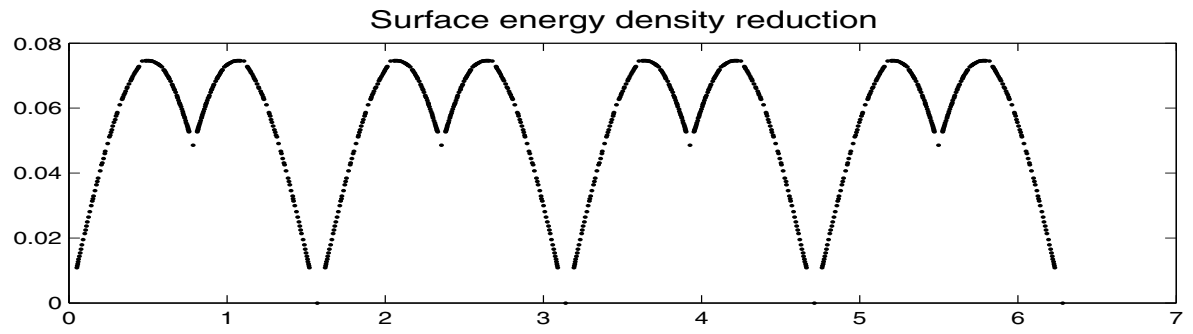

First difference quotients

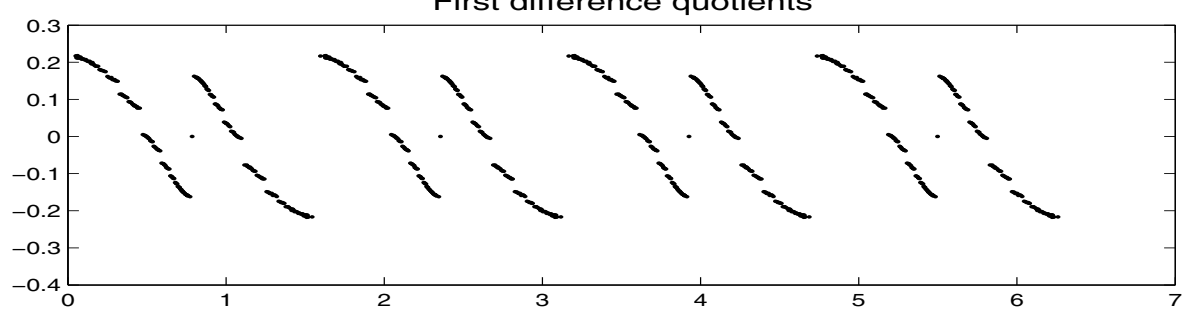

FIGURE 2. The function $W_{\mathrm{rel}}^{(s)}$ and a numerical approximation of $\frac{1}{\tau \tau} \nabla W_{\mathrm{rel}}^{s}(\nu) \cdot \tau$ evaluated on the set $\left\{\frac{\nu}{|\nu|} \mid \nu \in \mathbb{Z}^{2} \backslash\{0\}\right\} \subset S_{1}$. The horizontal axis is the angle $\alpha=\arctan \left(\nu_{2} / \nu_{1}\right)$.

An explicit estimate how quickly $\varepsilon$ converges to 0 as $\rho$ tends to infinity is not required. Ideally one would like to show that $\lim _{\rho \rightarrow \infty} \sigma_{\rho}$ admits an integral-representation if $\varepsilon$ does not depend on $\rho$, and study the precise properties of the surface energy density in a second step. This approach fails as currently there is no general method available which shows that the surface energies can be localized, i.e. written as a surface integral. In the context of non-convex homogenization theory powerful techniques have been developed which demonstrate that in very general situations the leading order term $E_{\text {bulk }}$ can be written as a bulk integral (see e.g. [5], Thm. 14.5) but it is not clear whether a similar method works also for surface energies. To circumvent this problem we do not separate the two steps and assume instead that the interaction potentials $V_{1}$ and $V_{\sqrt{2}}$ depend on $\rho$ in such a way that the number $\varepsilon$ (defined by (1.9)) satisfies $\varepsilon=o(1)$ as $\rho \rightarrow \infty$.

The key points are that $W_{\text {rel }}^{s}$ is independent of the domain $\Omega$ and that the surface energy can be written as an integral. The surface energy density is determined an exponentially decaying discrete boundary layer. This is analog to boundary layers for classical homogenization deriving from periodic oscillations on the scale of the heterogeneities. The boundary layers decay to a constant exponentially from the boundary when that is a rational plane, and their effect can be described by a suitable formula on functions which are periodic in the tangential directions, see [16] and [23], Chapter 18. In the discrete setting we obtain more detailed information by solving an algebraic Riccati equation. The solution characterizes the transfer operator whose powers deliver the boundary layer (Sect. 2).

The function $W_{\text {rel }}^{s}$ is $1 /|\tau|$ times the ground state energy in the idealized situation where the lattice is a semiinfinite strip with normal $\nu$ among maps $y$ which are periodic with period $\tau=\nu^{\perp}=\left(\begin{array}{cc}0 & -1 \\ 1 & 0\end{array}\right) \nu$, and $|\tau|$ denotes the length (Euclidean norm) of $\tau$.

A numerical visualization of $W_{\text {rel }}^{s}$ suggests that the function $\alpha \mapsto W_{\text {rel }}^{s}((\cos (\alpha), \sin (\alpha)))$ is not continuously differentiable, see Figure 2. In Section 4 the graph of $\nu \mapsto W_{\text {rel }}^{s}(\nu)$ is studied numerically. The results show that the surface relaxation pattern has a nontrivial effect on the Frank diagram, see Figure 4. In particular, it seems that the boundary of the Frank diagram is not differentiable at the (dense) set of those $\alpha$ where $\nu \in \mathbb{Z}^{2} \backslash\{0\}$. This effect is observed frequently in lattice-systems, $c f$. [2,8]. To the best knowledge of the author not even continuous dependency on $\alpha$ has yet been established. However, in the case of boundary layers in continuum homogenization recent results in this direction have been obtained in [14]. 
The proposed model can be extended in many different ways. Higher dimensional versions can be formulated and analyzed in an analogous fashion. An adaptation of the mathematical proofs involves a slight modification of the notation, but no new concepts.

It would be desirable to extend the analysis in the spirit of Gamma-convergence to continuum to deformations $\phi$. This amounts to a generalization of Theorem 1.2 to nontrivial atomistic deformation fields which satisfy the clamping condition only in a weak sense. It is this case where the importance of the admissibility condition $y \in \mathcal{A}$ becomes most obvious, because without it not even the correct bulk term can be recovered, $c f$. [12].

More realistic potentials involve long-range and non-pair interactions, $c f$. [10]. Although some crucial steps have been taken to adapt the existing methods to the variational framework [24], additional ideas are required to characterize surface corrections.

\subsection{A one-dimensional example}

We illustrate the fact that the $W_{\mathrm{cl}}^{s}$ overestimates the true surface energy $\sigma$ by the following, one-dimensional example. To avoid confusion we will use "end energy" instead of "surface energy" in this section. Let $V_{1}, V_{2} \in$ $C^{2}([0, \infty))$ be uniformly convex such that

$$
V_{1}^{\prime}(1)+2 V_{2}^{\prime}(2)=0
$$

and define $E_{\text {bulk }}:=V_{1}(1)+V_{2}(2)$. For each positive integer $\rho>0$ we define the end energy

$$
\begin{aligned}
\sigma_{\text {end }}^{(\rho)}:= & \min _{y(0) \leq \ldots \leq y(\rho)}\left\{\sum_{x=0}^{\rho-1} V_{1}(y(x+1)-y(x))+\sum_{x=1}^{\rho-1} V_{2}(y(x+1)-y(x-1))\right\} \\
& -(\rho+1) E_{\text {bulk }} .
\end{aligned}
$$

It is not hard to show that even without the extraction of subsequences the limit $\lim _{\rho \rightarrow \infty} \sigma_{\text {end }}^{(\rho)}=\sigma$ exists. We focus on models that have only weakly pronounced end layers and consider the potentials $V_{1}$ and $V_{2}$ that depend not only on the distance between particles but also on a parameter $\varepsilon=V_{1}^{\prime}(1)-2 V_{2}^{\prime}(2)$. The objective is to discuss the behavior of $\sigma$ as $\varepsilon \rightarrow 0$. In the one-dimensional case the first correction as $\varepsilon \rightarrow 0$ can be computed explicitly.

Proposition 1.5. A) Let $V_{1}$ and $V_{2}$ be two uniformly convex interaction potentials, i.e. there exists a number $\gamma>0$ such that $\min _{r} V_{i}^{\prime \prime}(r) \geq \gamma$ for $i \in\{1,2\}$ and assume that $V_{1}^{\prime}(1)+2 V_{2}^{\prime}(2)=0$. Then $\sigma=\lim _{\rho \rightarrow \infty} \sigma_{\text {end }}^{(\rho)}$ exists.

B) Assume furthermore that the $V_{1}$ and $V_{2}$, but not $\gamma$, depend on a small parameter $\varepsilon$ such that $\varepsilon=V_{1}^{\prime}(1)-2 V_{2}^{\prime}(2)$ and $V_{i} \in C^{2}\left((0, \infty) \times\left(-\varepsilon_{0}, \varepsilon_{0}\right)\right)$ for some $\varepsilon_{0}>0$. Let $K_{i}=\lim _{\varepsilon \rightarrow 0} V_{i}^{\prime \prime}(i)$ and $c_{i}=\lim _{\varepsilon \rightarrow 0} V_{i}(i)$, then

$$
\sigma=-\left(c_{1}+2 c_{2}\right)-\varepsilon^{2} \frac{1}{64 K_{2}}\left(\sqrt{1+4 K_{2} / K_{1}}-1\right)+o\left(\varepsilon^{2}\right) \text { if }|\varepsilon| \ll 1 .
$$

Proof. See appendix.

The leading order term of the end energy $\sigma$ is precisely the clamped end energy density $W_{\mathrm{cl}}^{s}$ (Def. 1.1; in fact there are two factors involved that cancel each other: the one-dimensional model has actually two ends with opposite normals and the formula for $W_{\mathrm{cl}}^{s}$ is derived from a model where each pair interaction is counted twice). The correction term quantifies the reduction of the end energy due to the formation of boundary layers. It is not very surprising that only local properties of the potentials $V_{1}$ and $V_{2}$ enter the formula since the assumption of strict convexity gives us a strong a priori estimate (6.2). 


\subsection{Sketch of the proof of Theorem $\mathbf{1 . 4}$}

The complete proof is given at the end of Section 3. One of the main achievements of the paper is to overcome several arising technical difficulties. Since the energy corrections are caused by the presence of surfaces we cannot use standard localization results (e.g. [5], Thm. 14.5) which work only for bulk problems.

We prove matching lower and upper bounds. For the upper bound we explicitly construct a sequence of admissible competitors which have the correct boundary layer. This construction will miss corner effects but as these energy contributions are only $O(1)$ and not $O(\rho)$ like the surface energy they do not affect the energy scale we are looking at. The construction is based on the solution of a simpler linear problem, the "cell-problem" in the homogenization literature. This cell problem is stated and solved in Proposition 2.1.

To prove the lower bound we show that as $\rho$ tends to infinity the displacements become so small that the linearization of the energy $E^{(\rho)}$ captures the essential energy contributions.

Due to the invariance under rigid body motions the energy is necessarily a nonconvex function of the atomic positions i.e. $E^{(\rho)}(\{y\})=E^{(\rho)}(\{R y\})$ for all $y: \mathbb{Z}^{2} \rightarrow \mathbb{R}^{2}$ and all $R \in S O(2)$. Therefore is not obvious why energy minimizers should be even roughly periodic in the tangential direction.

To overcome this difficulty we use rigidity estimates, $c f$. [13] which allow us to replace the non-convex energy by a convex quadratic energy. Rigidity estimates have been successfully applied to justify effective continuum models such as plate and shell theories [13] and more recently to extract linear theories from atomistic models [7,19-21]. The novel aspect in systems which are dominated by surfaces is that the limiting theory accounts for an exponentially decaying surface layer which depends on the normal vector. As a consequence we have to work with carefully constructed averages which respect the one-dimensional structure of the cell-problem. In particular, only tangential averages can be taken.

Since only integral norms can be controlled it is well possible that there are small patches in the crystal where the strains are big and therefore cannot be captured with a linear theory. In order to control the contribution of those bad patches we employ the standard method of removing the singular parts by multiplication with a suitable cutoff function. The key step consists in proving that resulting tangentially averaged bonds are still differences.

Throughout the proof the letter $C$ denotes a generic constant whose value can change from line to line but does not depend on $\rho$ or $\varepsilon$.

\section{The Limiting TheORY}

We derive an explicit formula for the function $W_{\text {rel }}^{s}$ which is based on an idealized situation where:

(1) the formerly non-quadratic pair-interaction potentials $V_{|\lambda|}(|\lambda+z|)$ are replaced by the quadratic approximation $V_{|\lambda|}(|\lambda|)+z \cdot\left(\frac{1}{2} A(\lambda) z+L(\lambda)\right)$ and

(2) the complicated set $\mathcal{L}^{(\rho)}$ is replaced by a family of half-spaces

$$
\mathbb{Z}_{\nu}^{2}:=\left\{x \in \mathbb{Z}^{2} \mid x \cdot \nu \leq 0\right\}
$$

The lattice vectors $\lambda$ and $\nu \in \mathbb{Z}^{2}$ denote the difference between two lattice points $\left(\lambda=x-x^{\prime}\right)$ and the outward pointing normal vectors associated to the sides of $\Omega$. Both $A(\lambda)$ and $L(\lambda)$ depend in a nontrivial way on $\lambda$. In many cases this dependency will not be shown explicitly and we write $A$ and $L$ instead of $A(\lambda)$ and $L(\lambda)$.

The inner product $(u, v)$ of two vectors $u, v \in \mathbb{R}^{d}$ is denoted by $u \cdot v$, the multiplication of a matrix $A$ with a vector $v$ is written $A v$ and consequentially $u \cdot A v$ is the same as $u^{T} A v$ in matrix notation.

For each pair $x, x^{\prime} \in \mathbb{Z}^{2}$ the vector $L\left(x-x^{\prime}\right) \in \mathbb{R}^{2}$ is given by

$$
L\left(x-x^{\prime}\right)=\left\{\begin{aligned}
\frac{1}{2}\left(x-x^{\prime}\right) & \text { if }\left|x-x^{\prime}\right|=1, \\
\frac{1}{4}\left(x^{\prime}-x\right) & \text { if }\left|x-x^{\prime}\right|=\sqrt{2} \\
0 & \text { else }
\end{aligned}\right.
$$


and the matrix $A\left(x-x^{\prime}\right) \in \mathbb{R}^{2 \times 2}$ by

$$
A\left(x-x^{\prime}\right)=\left\{\begin{aligned}
K_{1}\left(x-x^{\prime}\right) \otimes\left(x-x^{\prime}\right) & \text { if }\left|x-x^{\prime}\right|=1 \\
\frac{1}{2} K_{2}\left(x-x^{\prime}\right) \otimes\left(x-x^{\prime}\right) & \text { if }\left|x-x^{\prime}\right|=\sqrt{2} \\
0 & \text { else. }
\end{aligned}\right.
$$

We define the boundary of $\mathbb{Z}_{\nu}^{2}$ by

$$
\partial \mathbb{Z}_{\nu}^{2}=\left\{x \in \mathbb{Z}_{\nu}^{2} \mid \#\left\{x^{\prime} \in \mathbb{Z}_{\nu}^{2}|| x^{\prime}-x \mid \leq \sqrt{2}\right\}<9\right\},
$$

and the forces

$$
b_{\nu}(x):=\left\{\begin{aligned}
\sum_{x^{\prime} \in \partial \mathbb{Z}_{\nu}^{2}} v_{\nu}\left(x, x^{\prime}\right) L\left(x-x^{\prime}\right) & \text { if } x \in \partial \mathbb{Z}_{\nu}^{2}, \\
0 & \text { else }
\end{aligned}\right.
$$

where the weights $v_{\nu} \in\left\{0, \frac{1}{2}, 1\right\}$ are given by

$$
v_{\nu}\left(x, x^{\prime}\right)= \begin{cases}1 & \text { if }\left|x-x^{\prime}\right|=\sqrt{2} \\ \frac{1}{2} & \text { if }\left|x-x^{\prime}\right|=1 \\ 0 & \text { else }\end{cases}
$$

The weights account for cancellation effects, the motivation will become clear in Section 3. Here we only use the fact that the mean force is zero, i.e.

$$
\sum_{x \in \mathbb{Z}_{\nu}^{2}} b_{\nu}(x)=0 .
$$

This follows immediately from the symmetry of the weights $v_{\nu}$ :

$$
\begin{aligned}
\sum_{x \in \mathbb{Z}_{\nu}^{2}} b_{\nu}(x) & =\sum_{x \in \partial \mathbb{Z}_{\nu}^{2} \bmod \tau} \sum_{x^{\prime} \in \partial \mathbb{Z}_{\nu}^{2}} v_{\nu}\left(x, x^{\prime}\right) L\left(x-x^{\prime}\right)=-\sum_{x^{\prime} \in \mathbb{Z}_{\nu}^{2} \bmod \tau} \sum_{x \in \mathbb{Z}_{\nu}^{2}} v_{\nu}\left(x^{\prime}, x\right) L\left(x^{\prime}-x\right) \\
& =-\sum_{x \in \mathbb{Z}_{\nu}^{2}} b_{\nu}(x)
\end{aligned}
$$

which implies (2.6).

For a $\tau$-periodic displacement function $z: \mathbb{Z}_{\nu}^{2} \rightarrow \mathbb{R}^{2}$ we consider the quadratic cost functional

$$
Q_{\nu}(z):=\sum_{x \in \mathbb{Z}_{\nu}^{2} \bmod \tau}\left\{b_{\nu}(x) \cdot z(x)+\frac{1}{4} \sum_{x^{\prime} \in \mathbb{Z}_{\nu}^{2}}\left(z(x)-z\left(x^{\prime}\right)\right) \cdot A\left(z(x)-z\left(x^{\prime}\right)\right)\right\} .
$$

The factor $\frac{1}{4}$ enters because we have to compensate for double-counting.

The following theorem asserts that the minimum of $Q_{\nu}$ can be found by solving a finite-dimensional nonlinear matrix equation.

Theorem 2.1. For each $\nu \in \mathbb{Z}^{2} \backslash\{0\}$ :

A: There exists a symmetric, positive semi-definite matrix $H_{\nu} \in \mathbb{R}^{2 d \times 2 d}$, where $d=\#\left(\partial \mathbb{Z}_{\nu}^{2} \bmod \tau\right)=$ $|\nu|_{1}=\left|\nu_{1}\right|+\left|\nu_{2}\right|$, that solves the discrete algebraic Riccati-equation

$$
-\left(H+N^{T}\right)(M+H)^{-1}(H+N)+P=0
$$

with the $2 d \times 2 d$-matrices $M, N$ and $P$ given by $(2.23)-(2.25)$. 
B: The minimum of $Q_{\nu}(\cdot)$ is given by $-\frac{1}{2} b_{\nu} \cdot \widehat{H}_{\nu} b_{\nu}$ where $\widehat{H}_{\nu}$ is the Moore-Penrose inverse of $H_{\nu}$. The minimum energy is achieved by

$$
z_{\nu}\left(x-k e_{12}\right)=\left((\operatorname{Id}+\Lambda)^{k} g_{\nu}\right)(x) \quad \text { if } x \in \mathbb{Z}_{\nu}^{2}(0), \quad e_{12}=e_{1}+e_{2},
$$

where $e_{1}$ and $e_{2}$ are the standard unit-vectors, $\Lambda=-(M+H)^{-1}(H+N)$ and

$$
g_{\nu}=\widehat{H}_{\nu} b_{\nu}
$$

There exists $C>0$ and $z_{\nu}^{\infty} \in \mathbb{R}^{2}$ such that $z_{\nu}$ satisfies the exponential decay estimate

$$
\left|z_{\nu}(x)-z_{\nu}^{\infty}\right| \leq C \exp \left(\frac{1}{C} \nu \cdot x\right) \text { for all } x \in \mathbb{Z}_{\nu}^{2} .
$$

Remark 2.2. In classical (linear and continuous) homogenization it is known that boundary layers deriving from periodic oscillations on the scale of the heterogeneities decay to a constant exponentially from the boundary when that is a "rational" plane, and their effect can be described by a suitable formula on functions which are periodic in the tangential directions, see e.g. [16], Theorem 1.10.1. However, since the discrete model differs in several ways from linear continuum problems, a generalization of the result to the discrete problem is not immediate.

The question whether (2.1) admits more than one symmetric nonnegative solution is beyond the scope of this paper, see however [15]. The linearized energy $Q_{\nu}$ has inherited the invariance under translations from the original energy $E^{(\mathcal{L})}$. This implies that the kernel of $H$ contains the two-dimensional vectorspace consisting of all translations

$$
\mathcal{V}=\left\{z: \partial \mathbb{Z}_{\nu}^{2} \rightarrow \mathbb{R}^{2} \mid z(x)=t \text { where } t \in \mathbb{R}^{2} \text { is independent of } x\right\}
$$

and in particular the classical inverse of $H$ does not exist.

Definition 2.3. Let $\nu, \widehat{H}_{\nu}$ and $b$ be defined as in Theorem 2.1. For each $\nu \in \mathbb{Z}^{2} \backslash\{0\}$ we define

$$
W_{\mathrm{rel}}^{s}(\nu):=-\frac{1}{2|\nu|} b_{\nu} \cdot \widehat{H}_{\nu} b_{\nu} .
$$

Proof of Theorem 2.1. We exploit the one-dimensional structure and apply standard ideas from Calculus of Variations.

Obviously the infimum of $Q_{\nu}$ is smaller or equal to 0 since $z(x) \equiv 0$ is an element of $X_{\nu}$. We will demonstrate first that $Q_{\nu}$ is bounded from below, i.e. there exists a constant $C>0$ which depends only on $L, A$ and $\nu$ such that

$$
Q_{\nu}(z) \geq-C
$$

for all $z$. To obtain this bound it suffices to show that the quadratic part of $Q_{\nu}$ is coercive, i.e. there exists a constant $C>0$ such that

$$
\mathcal{D}_{\text {discr }}(z) \leq C \mathcal{E}_{\text {discr }}(z),
$$

where

$$
\begin{aligned}
& \mathcal{D}_{\text {discr }}(z)=\sum_{x \in \mathbb{Z}_{\nu}^{2} \bmod \tau} \sum_{\substack{x^{\prime} \in \mathbb{Z}_{\nu}^{2} \\
\left|x-x^{\prime}\right| \leq \sqrt{2}}}\left|z(x)-z\left(x^{\prime}\right)\right|^{2}, \\
& \mathcal{E}_{\text {discr }}(z)=\sum_{x \in \mathbb{Z}_{\nu}^{2} \bmod \tau} \sum_{\substack{x^{\prime} \in \mathbb{Z}_{\nu}^{2} \\
\left|x-x^{\prime}\right| \leq \sqrt{2}}}\left(\left(z(x)-z\left(x^{\prime}\right)\right) \cdot\left(x-x^{\prime}\right)\right)^{2} .
\end{aligned}
$$


Indeed,

$$
\begin{array}{r}
\sum_{x \in \partial \mathbb{Z}_{\nu}^{2} \bmod \tau} b_{\nu}(x) \cdot z(x)=\sum_{x \in \partial \mathbb{Z}_{\nu}^{2} \bmod \tau} \sum_{x^{\prime} \in \partial \mathbb{Z}_{\nu}^{2}} v_{\nu}\left(x, x^{\prime}\right) L\left(x-x^{\prime}\right) \cdot z(x) \\
=\frac{1}{2} \sum_{x \in \partial \mathbb{Z}_{\nu}^{2} \bmod \tau} \sum_{x^{\prime} \in \partial \mathbb{Z}_{\nu}^{2}} v_{\nu}\left(x, x^{\prime}\right) L\left(x-x^{\prime}\right) \cdot\left(z(x)-z\left(x^{\prime}\right)\right) \geq-\frac{1}{2} \sqrt{C \mathcal{D}_{\text {discr }}(z)},
\end{array}
$$

by Cauchy-Schwarz if

$$
C \geq \sum_{x \in \partial \mathbb{Z}_{\nu}^{2} \bmod \tau} \sum_{\substack{x^{\prime} \in \partial \mathbb{Z}_{\nu}^{2} \\\left|x-x^{\prime}\right| \leq \sqrt{2}}} L\left(x-x^{\prime}\right)^{2} .
$$

Thanks to the structure of the matrices $A\left(x-x^{\prime}\right)$ one finds that

$$
\mathcal{E}_{\text {discr }}(z) \leq C \sum_{x \in \mathbb{Z}_{\nu}^{2} \bmod } \sum_{\tau x^{\prime} \in \mathbb{Z}_{\nu}^{2}}\left(z(x)-z\left(x^{\prime}\right)\right) \cdot A\left(z(x)-z\left(x^{\prime}\right)\right),
$$

and (2.15) yields that

$$
Q_{\nu}(z) \geq \frac{1}{C} \mathcal{D}_{\text {discr }}(z)-C \sqrt{\mathcal{D}_{\text {discr }}(z)} .
$$

Hence, $Q_{\nu}(z) \geq-\frac{1}{4} C$, and the a priori bound (2.14) has been established.

To prove (2.15) we have to overcome the technical difficulty that we only control certain projections of the differences, namely $\left|\left(x-x^{\prime}\right) \cdot\left(z(x)-z\left(x^{\prime}\right)\right)\right|^{2}$ but would like to control the full difference $\left|z(x)-z\left(x^{\prime}\right)\right|^{2}$. For example, the displacement function $z^{(\alpha)}(x)=\left(\begin{array}{cc}0 & \alpha \\ -\alpha & 0\end{array}\right) x$ has the property that for every $\alpha \in \mathbb{R}$ and every $x, x^{\prime}$ the equality $\left(z^{(\alpha)}(x)-z^{(\alpha)}\left(x^{\prime}\right)\right) \cdot A\left(z^{(\alpha)}(x)-z^{(\alpha)}\left(x^{\prime}\right)\right)=0$ holds. Hence, the proof of (2.15) involves more than the algebraic properties of the matrices $A\left(x-x^{\prime}\right)$, in addition we have to use the periodic boundary condition.

For any open set $S \subset \mathbb{R}^{2}$ and a function $u \in H^{1}\left(S, \mathbb{R}^{2}\right)$ the quadratic forms $\mathcal{D}$ and $\mathcal{E}$ are given by

$$
\begin{aligned}
& \mathcal{D}(S, u)=\sum_{i, j=1}^{2} \int_{S}\left(\frac{\partial u_{i}}{\partial \eta_{j}}\right)^{2} \mathrm{~d} \eta \\
& \mathcal{E}(S, u)=\sum_{i, j=1}^{2} \int_{S}\left(\frac{\partial u_{i}}{\partial \eta_{j}}+\frac{\partial u_{j}}{\partial \eta_{i}}\right)^{2} \mathrm{~d} \eta .
\end{aligned}
$$

Define the semi-infinite strip

$$
\begin{aligned}
U= & \left(\bigcup_{\substack{x \in \mathbb{Z}_{\nu}^{2} \\
x+e_{1}, x+e_{2} \in \mathbb{Z}_{\nu}^{2}}} \operatorname{conv}\left(\left\{x, x+e_{1}, x+e_{2}\right\}\right)\right) \\
& \bigcup\left(\bigcup_{\substack{x \in \mathbb{Z}_{\nu}^{2} \\
x-e_{1}, x-e_{2} \in \mathbb{Z}_{\nu}^{2}}} \operatorname{conv}\left(\left\{x, x-e_{1}, x-e_{2}\right\}\right)\right) \bmod \tau
\end{aligned}
$$

and the bounded domain

$$
U_{0}=U \backslash(U-\nu)
$$

As $U_{0}$ is a bounded Lipschitz-domain here exists a constant $C$ such that every $\tau$-periodic function $u \in H^{1}\left(U_{0}, \mathbb{R}^{2}\right)$ satisfies Korn's inequality

$$
\mathcal{D}\left(U_{0}, u\right) \leq C \mathcal{E}\left(U_{0}, u\right)
$$

see e.g. [17]. Since $U$ can be written as the disjoint union of translated copies of $U_{0}$

$$
U=\cup_{k \in \mathbb{Z}, k \geq 0} U_{k}
$$


with $U_{k}=U_{0}-k \nu$ we find that for every $u \in H^{1}(U)$

$$
\mathcal{D}(U, u)=\sum_{k=0}^{\infty} \mathcal{D}\left(U_{k}, u\right) \leq C \sum_{k=0}^{\infty} \mathcal{E}\left(U_{k}, u\right)=\mathcal{E}(U, u)
$$

and thus the Korn-constant of the unbounded domain $U$ is bounded from above by the Korn-constant of $U_{0}$.

Define now for each $\tau$-periodic map $z: \mathbb{Z}_{\nu}^{2} \rightarrow \mathbb{R}^{2}$ the piecewise affine interpolation $u: U \rightarrow \mathbb{R}^{2}$ which is affine on each triangle $\operatorname{conv}(T)$ where $T=\left\{x, x+e_{1}, x+e_{2}\right\}$ or $T=\left\{x, x-e_{1}, x-e_{2}\right\}$ for some $x \in \mathbb{Z}_{\nu}^{2}$. We will demonstrate now that there exists a universal constant $C$ such that the function $u$ satisfies two simple estimates:

$$
\begin{gathered}
\mathcal{E}(U, u) \leq C \sum_{x \in \mathbb{Z}_{\nu}^{2} \bmod \tau} \sum_{\substack{x^{\prime} \in \mathbb{E}_{\nu}^{2} \\
\left|x-x^{\prime}\right| \leq \sqrt{2}}}\left|\left(z(x)-z\left(x^{\prime}\right)\right) \cdot\left(x-x^{\prime}\right)\right|^{2} \\
\sum_{x \in \mathbb{Z}_{\nu}^{2} \bmod \tau} \sum_{\substack{x^{\prime} \in \mathbb{Z}_{\nu}^{2} \\
\left|x-x^{\prime}\right| \leq \sqrt{2},}}\left|z(x)-z\left(x^{\prime}\right)\right|^{2} \leq C \mathcal{D}(U, u) .
\end{gathered}
$$

We consider one triangle $T \subset \mathbb{Z}_{\nu}^{2}$ and assume first that $T=\left\{x, x+e_{1}, x+e_{2}\right\}$.

$$
\begin{aligned}
\mathcal{E}(\operatorname{conv}(T), u)= & 2\left(\left(z\left(x+e_{1}\right)-z(x)\right) \cdot e_{1}\right)^{2}+2\left(\left(z\left(x+e_{2}\right)-z(x)\right) \cdot e_{2}\right)^{2} \\
& +\left(\left(z\left(x+e_{1}\right)-z(x)\right) \cdot e_{2}+\left(z\left(x+e_{2}\right)-z(x)\right) \cdot e_{1}\right)^{2} \\
\leq & 5\left(\left(z\left(x+e_{1}\right)-z(x)\right) \cdot e_{1}\right)^{2}+5\left(\left(z\left(x+e_{2}\right)-z(x)\right) \cdot e_{2}\right)^{2} \\
& +3\left(\left(z\left(x+e_{1}\right)-z\left(x+e_{2}\right)\right) \cdot\left(e_{1}-e_{2}\right)\right)^{2} .
\end{aligned}
$$

In the case $T=\left\{x, x-e_{1}, x-e_{2}\right\}$ we obtain the analogous estimate. Considering the union of the triangles delivers (2.18) since each bond occurs at most twice. The proof of inequality (2.19) is similar.

The discrete version of Korn's inequality (2.17) is now a direct consequence of (2.19), (2.17) and (2.18). Thus, we have established that the a priori estimate (2.14) indeed holds.

Now we will demonstrate that there exists a non-negative solution $H_{\nu}$ of the Riccati equation (2.8) such

$$
\min _{g}\left\{\frac{1}{2} g H g+b \cdot g\right\}=\inf _{z} Q(z) .
$$

The $k$-th layer $\mathbb{Z}_{\nu}^{2}(k)$ of $\mathbb{Z}_{\nu}^{2}$ is defined by $\mathbb{Z}_{\nu}^{2}(k):=\partial \mathbb{Z}_{\nu}^{2}-k e_{12}$, and maps $z: \partial \mathbb{Z}_{\nu}^{2} \bmod \tau \rightarrow \mathbb{R}^{2}$ are identified with vectors $q \in \mathbb{R}^{2 d}, d=\#\left(\partial \mathbb{Z}_{\nu}^{2} \bmod \tau\right)$. From now on we assume without loss of generality that $0 \leq \nu \cdot e_{1} \leq \nu \cdot e_{2}$; it can be checked that

$$
\mathbb{Z}_{\nu}^{2}=\dot{\cup}_{k \in \mathbb{N}} \mathbb{Z}_{\nu}^{2}(k)
$$

We rearrange the terms in (2.7) slightly in order to distinguish between interactions within layers $\left(\mathbb{Z}_{\nu}^{2}(k)\right)$ and between successive layers $\left(\mathbb{Z}_{\nu}^{2}(k)\right.$ and $\left.\mathbb{Z}_{\nu}^{2}(k+1)\right)$ :

$$
\begin{aligned}
Q_{\nu}(z)= & \sum_{x \in \partial \mathbb{Z}_{\nu}^{2} \bmod \tau} b_{\nu}(x) \cdot z(x) \\
& +\frac{1}{2} \sum_{k=0}^{\infty} \sum_{x \in \partial \mathbb{Z}_{\nu}^{2}(k) \bmod \tau}\left(\frac{1}{2} \sum_{x^{\prime} \in \partial \mathbb{Z}_{\nu}^{2}(k)}\left(z(x)-z\left(x^{\prime}\right)\right) \cdot A\left(z(x)-z\left(x^{\prime}\right)\right)\right. \\
& \left.+\sum_{x^{\prime} \in \partial \mathbb{Z}_{\nu}^{2}(k+1)}\left(z(x)-z\left(x^{\prime}\right)\right) \cdot A\left(z(x)-z\left(x^{\prime}\right)\right)\right),
\end{aligned}
$$


where $A=A\left(x-x^{\prime}\right)$. Let $q_{k}(x):=z\left(x-k e_{12}\right)$ where $x \in \partial \mathbb{Z}_{\nu}^{2}$ and $k \in \mathbb{N} \cup\{0\}$ and construct matrices $M, N, P \in \mathbb{R}^{2 d \times 2 d}$ such that

$$
Q_{\nu}(z)=b_{\nu} \cdot q_{0}+\frac{1}{2} \sum_{k=0}^{\infty}\left(\begin{array}{c}
q_{k+1}-q_{k} \\
q_{k}
\end{array}\right) \cdot\left(\begin{array}{cc}
M & N \\
N^{T} & P
\end{array}\right)\left(\begin{array}{c}
q_{k+1}-q_{k} \\
q_{k}
\end{array}\right) .
$$

Interactions across the boundary between two neighboring layers are governed by $M$ :

$$
p \cdot M p:=\sum_{x \in \partial \mathbb{Z}_{\nu}^{2} \bmod \tau} \sum_{x^{\prime} \in \partial \mathbb{Z}_{\nu}^{2}} p\left(x^{\prime}\right) \cdot A\left(x-x^{\prime}+e_{12}\right) p\left(x^{\prime}\right) .
$$

Interactions between one layer and the differences between two neighboring layers:

$$
p \cdot N q:=\sum_{x \in \partial \mathbb{Z}_{\nu}^{2} \bmod \tau} \sum_{x^{\prime} \in \partial \mathbb{Z}_{\nu}^{2}}\left(q(x)-q\left(x^{\prime}\right)\right) \cdot A\left(x-x^{\prime}+e_{12}\right) p\left(x^{\prime}\right) .
$$

Interactions within one layer:

$$
\begin{aligned}
q \cdot P q:=\frac{1}{2} \sum_{x \in \partial \mathbb{Z}_{\nu}^{2} \bmod \tau} \sum_{x^{\prime} \in \partial \mathbb{Z}_{\nu}^{2}}\left[\left(q(x)-q\left(x^{\prime}\right)\right) \cdot A(x-\right. & \left.x^{\prime}\right)\left(q(x)-q\left(x^{\prime}\right)\right) \\
& \left.+2\left(q(x)-q\left(x^{\prime}\right)\right) \cdot A\left(x-x^{\prime}+e_{12}\right)\left(q(x)-q\left(x^{\prime}\right)\right)\right] .
\end{aligned}
$$

Now we are in a position to show that the discrete algebraic Riccati equation (2.8) has a solution $H$ which satisfies (2.20). First we define

$$
J_{n}\left(\left\{q_{k}\right\}_{k=0 \ldots n}\right)=\frac{1}{2} \sum_{k=0}^{n-1}\left(\begin{array}{c}
q_{k+1}-q_{k} \\
q_{k}
\end{array}\right) \cdot\left(\begin{array}{cc}
M & N \\
N^{T} & P
\end{array}\right)\left(\begin{array}{c}
q_{k+1}-q_{k} \\
q_{k}
\end{array}\right) .
$$

Note that due to the non-negativity of $A$ the matrix $\left(\begin{array}{cc}M & N \\ N^{T} & P\end{array}\right)$ is non-negative and thus $J_{n}$ is convex and non-negative. Moreover, range $(N) \subseteq \operatorname{range}(M)$, this implies that for every non-negative symmetric matrix $H$ the matrix $(M+H)^{-1}(H+N)$ is well defined within the range of $M+H$. The matrix $H_{n}$ is defined via the iteration

$$
H_{n+1}=H_{n}+P-\left(H_{n}+N\right)^{T}\left(M+H_{n}\right)^{-1}\left(H_{n}+N\right), \quad H_{0}=0 .
$$

We will show next that $H_{n}$ is non-negative and bounded by establishing that

$$
\frac{1}{2} g \cdot H_{n} g=\min \left\{J_{n}\left(\left\{q_{k}\right\}_{k=0 \ldots n}\right) \mid q_{0}=g\right\} .
$$

This result is an direct generalization of Lemma 8.2.1 in [22]. It can be checked using (2.26) that the following algebraic identity holds

$$
\begin{aligned}
& \left(q_{k+1}-q_{k}+\left(H_{n-k-1}+M\right)^{-1}\left(H_{n-k-1}+N\right) q_{k}\right) \\
& \cdot\left(H_{n-k-1}+M\right)\left(q_{k+1}-q_{k}+\left(H_{n-k-1}+M\right)^{-1}\left(H_{n-k-1}+N\right) q_{k}\right) \\
= & \left(q_{k+1}-q_{k}\right) \cdot M\left(q_{k+1}-q_{k}\right)+2\left(q_{k+1}-q_{k}\right) \cdot N q_{k}+q_{k} \cdot P q_{k} \\
& -q_{k} \cdot H_{n-k-1} q_{k}+q_{k+1} \cdot H_{n-k-1} q_{k+1} .
\end{aligned}
$$


Summation over $k$ together with the initial condition $H_{0}=0$ yields the identity

$$
\begin{aligned}
J_{n}\left(\left\{q_{k}\right\}_{k=0 \ldots n}\right)= & \frac{1}{2}\left(g \cdot H_{n} g+\sum_{k=0}^{n-1}\left(q_{k+1}-q_{k}+\left(H_{n-k-1}+M\right)^{-1}\left(H_{n-k-1}+N\right) q_{k}\right)\right. \\
& \left.\cdot\left(H_{n-k-1}+M\right)\left(q_{k+1}-q_{k}+\left(H_{n-k-1}+M\right)^{-1}\left(H_{n-k-1}+N\right) q_{k}\right)\right),
\end{aligned}
$$

where $g=q_{0}$. The choice $q_{k+1}=q_{k}-\left(H_{n-k-1}+M\right)^{-1}\left(H_{n-k-1}+N\right) q_{k}, q_{0}=g$ shows that equation (2.27) is correct. As each term in the definition of $J_{n}$ is non-negative $H_{n}$ is non-decreasing with $n$ and since

$$
q_{k}= \begin{cases}g & \text { if } k=0 \\ 0 & \text { else }\end{cases}
$$

is admissible $H_{n}$ is bounded. Hence, the limit $H=\lim _{n \rightarrow \infty} H_{n}$ exists, has the property

$$
\frac{1}{2} g \cdot H g=\inf \left\{J_{\infty}\left(\left\{q_{k}\right\}_{k=0 \ldots \infty}\right) \mid q_{0}=g\right\}
$$

and solves the discrete algebraic Riccati equation (2.8). A minimizer of $J_{\infty}$ is given by $q_{k}=(\mathrm{Id}-\Lambda)^{k} g$, $\Lambda=(H+M)^{-1}(H+N)$. Moreover,

$$
\min _{g}\left(\frac{1}{2} g \cdot H_{\nu} g+b_{\nu} \cdot g\right)=\inf _{z} Q_{\nu}(z)
$$

To see that $\min _{g}\left(\frac{1}{2} g \cdot H_{\nu} g+b_{\nu} \cdot g\right)=-\frac{1}{2} b_{\nu} \cdot \widehat{H_{\nu}} b_{\nu}$ we first recall that the Moore-Penrose inverse $\widehat{H}$ of $H$ is characterized by the equations

$$
H \widehat{H} H=H, \widehat{H} H \widehat{H}=\widehat{H}, H \widehat{H}=\widehat{H} H .
$$

Estimate (2.14) implies that $\inf _{g}\left(b_{\nu} \cdot g+\frac{1}{2} g \cdot H g\right)>-\infty$ and therefore $b_{\nu} \in(\text { ker } H)^{\perp}$ or equivalently $b_{\nu}=H \widehat{H} b_{\nu}$. Thanks to this formula we have the identity

$$
\frac{1}{2} g \cdot H_{\nu} g+g \cdot b_{\nu}=\frac{1}{2}((g+\widehat{H} b) \cdot H(g+\widehat{H} b)-b \cdot \widehat{H} b)
$$

and the minimum is achieved for $g=-\widehat{H} b$. Hence,

$$
\inf _{z} Q_{\nu}(z)=-\frac{1}{2|\nu|} b_{\nu} \cdot \widehat{H_{\nu}} b_{\nu}
$$

Finally, we prove the exponential decay estimate (2.11). First note that the dimension of the kernel of $H$ is at least two, since translations leave the values of $J_{n}$ and $J_{\infty}$ unchanged. It can be checked by inspection that the translations are also contained in the kernels of $M$ and $N$, this implies that the translations are contained in the kernel of $\Lambda$. Let $\left(\lambda_{i}, \pi_{i}\right)_{i=1 \ldots m}$ be the eigenvalues and projections on the generalized eigenspaces of $\Lambda$ so that $\lambda_{1}=0$. Since $\mathcal{D}_{\text {discr }}\left(z_{\nu}\right)$ is finite either $\left|1+\lambda_{i}\right|<1$ or $\pi_{i} g=0$ for all $i>1$, and thus (2.11) holds.

\section{JustificAtion OF THE Limiting THEORY}

The basic idea is that if the spring tensions become small, the accuracy of the quadratic approximation (2.22) improves and by convexity ground states are more or less periodic in the $\tau$-direction. However, spatial oscillations in the normal direction will always be present although the relaxation pattern becomes rapidly small with growing distance from the surface. A visualization of a typical energy minimizer can be seen in Figure 5 . 
The justification is based on two steps. First we prove a rigidity result for the discrete gradients and justify the linearized theory based on this bound. The second ingredient of Theorem 2.1, the periodicity in the $\tau$-direction, follows from a weak convergence argument and the existence of minimizers within the set of periodic configurations which close to the identity map.

\subsection{Rigidity estimates}

The main tool is a rigidity estimate for continuous deformations.

Theorem 3.1 ([13]). Let $\Omega \subset \mathbb{R}^{2}$ be a simply connected domain with Lipschitz-boundary. There exists a constant $C(\Omega)>0$ with the property that for all $u \in W^{1,2}\left(\Omega, \mathbb{R}^{2}\right)$ there exist $R \in S O(2)$ such that the inequality

$$
\|\nabla u-R\|_{L^{2}(\Omega)}^{2} \leq C \int_{\Omega} \operatorname{dist}(\nabla u(x), S O(2))^{2} \mathrm{~d} x
$$

holds. The constant $C$ is invariant under rescaling, i.e. $C(\rho \Omega)=C(\Omega)$ for all $\rho \in(0, \infty)$.

We say that a function $W: \mathbb{R}^{2 \times 4} \rightarrow \mathbb{R}$ which is invariant under $S O(2)$ in the sense that $W(R F)=W(F)$ for all $R \in S O(2), F \in \mathbb{R}^{2 \times n}$ has single-well structure if the set of minima consists of precisely one orbit of $S O(2)$. The map $y: Q \cap \mathbb{Z}^{2} \rightarrow \mathbb{R}^{2}$ is admissible if $\operatorname{det}\left(y\left(x_{2}\right)-y\left(x_{1}\right), y\left(x_{3}\right)-y\left(x_{1}\right)\right) \operatorname{det}\left(x_{2}-x_{1}, x_{3}-x_{1}\right) \geq 0$ for all $x_{1}, x_{2}, x_{3} \in \mathbb{Z}^{2} \cap Q$, where $Q=[0,1]^{2}$ is the two-dimensional unit-square.

Lemma 3.2. Let $V_{1}$ and $V_{\sqrt{2}}$ satisfy the assumptions (1.2)-(1.4) and recall the definition of $\varepsilon$ in equation (1.9). There exist two constants $C$ and $\varepsilon_{0}>0$ that depend only on $V_{1}$ and $V_{\sqrt{2}}$ such that for any $y: Q \cap \mathbb{Z}^{2} \rightarrow \mathbb{R}^{2}$, $\varepsilon \in\left(0, \varepsilon_{0}\right)$

$$
\int_{Q} \operatorname{dist}\left(\nabla u^{(y)}, S O(2)\right)^{2} \leq C W_{\text {cell }}\left(y(0), y\left(e_{1}\right), y\left(e_{1}+e_{2}\right), y\left(e_{2}\right)\right)
$$

where the map $u^{(y)}$ is the interpolation between the values of $y$ which is affine on each triangle $\operatorname{conv}\left\{0, e_{1}, e_{2}\right\}$, $\operatorname{conv}\left\{e_{1}, e_{2}, e_{1}+e_{2}\right\}$ and

$W_{\text {cell }}\left(y_{1}, y_{2}, y_{3}, y_{4}\right):=\left\{\begin{array}{r}\frac{1}{2} \sum_{i=1}^{4}\left(V_{1}\left(\left|y_{i+1 \bmod 4}-y_{i}\right|\right)+V_{\sqrt{2}}\left(\left|y_{i+2 \bmod 4}-y_{i}\right|\right)\right)-W_{\mathrm{cl}}(\mathrm{Id}) \quad \text { if } y \text { is admissible, } \\ +\infty \text { if } y \text { is not admissible. }\end{array}\right.$

Note that each of the terms $V_{\sqrt{2}}$ in the definition of $W_{\text {cell }}$ appears twice.

Proof of Lemma 3.2. Define the reference function

$$
W_{\text {ref }}\left(y_{1}, y_{2}, y_{3}, y_{4}\right):=\int_{Q}\left|\nabla u^{(y)}-S O(2)\right|^{2}
$$

Both functions $W_{\text {ref }}$ and $W_{\text {cell }}$ are invariant under translations and rotations, i.e. $W_{\text {cell }}(F)=W_{\text {cell }}(R F+v)$ and $W_{\text {ref }}(F)=W_{\text {ref }}(R F+v)$ for all $R \in S O(2), F \in \mathbb{R}^{2 \times 4}, v \in \mathbb{R}^{2} \otimes(1,1,1,1)$. To remove this degeneracy we introduce the set

$$
\mathcal{N}:=\operatorname{span}\left\{\left(\begin{array}{l}
1 \\
0
\end{array}\right) \otimes(1,1,1,1),\left(\begin{array}{l}
0 \\
1
\end{array}\right) \otimes(1,1,1,1),\left(\left(\begin{array}{c}
1 \\
-1
\end{array}\right),\left(\begin{array}{c}
1 \\
1
\end{array}\right),\left(\begin{array}{c}
-1 \\
1
\end{array}\right),\left(\begin{array}{c}
-1 \\
-1
\end{array}\right)\right)\right\}
$$

and observe that it suffices to prove the lemma for $\left(y_{0}, y_{1}, y_{2}, y_{3}\right)=F \in \mathcal{N}^{\perp}$. Indeed, for general matrices $F$ we can define a translation $v_{i}:=\frac{1}{4} \sum_{j=1}^{4} F_{i j} \otimes(1,1,1,1)$. Since $-\mathrm{Id} \in S O(2)$, the connectedness of $S O(2)$ together with the intermediate value theorem implies the existence of $R \in S O(2)$ such that $R(F-v) \in \mathcal{N}^{\perp}$. 


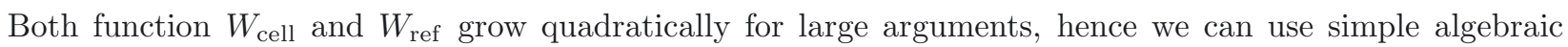
estimates to show that there exists $K, C>0$ such that (3.2) holds if $|F|>K$, thus it is enough to consider the compact set $|F| \leq K$.

Set $\widetilde{I d}:=\frac{1}{2}\left(\left(\begin{array}{c}-1 \\ -1\end{array}\right),\left(\begin{array}{c}1 \\ -1\end{array}\right),\left(\begin{array}{l}1 \\ 1\end{array}\right),\left(\begin{array}{c}-1 \\ 1\end{array}\right)\right)$, this matrix takes the role of the identity matrix in the context of cell deformations. It can be checked by an explicit calculation that $\widetilde{\text { Id }}$ is stationary, i.e. $\nabla W_{\text {cell }}( \pm \widetilde{\mathrm{Id}})=0$. Furthermore, since $W_{\text {ref }}( \pm \widetilde{\text { Id }})=0$ and $S O(2) \widetilde{\text { Id }} \cap \mathcal{N}^{\perp}=\{ \pm \widetilde{\text { Id }}\}$ with nonzero intersection angle we have that $|F-\{ \pm \widetilde{\mathrm{Id}}\}|^{2} \leq C W_{\text {ref }}(F)$ if $F \in \mathcal{N}^{\perp},|F-\{ \pm \widetilde{\mathrm{Id}}\}| \leq \frac{1}{K}$ if $C$ is sufficiently large. Another explicit calculation shows that the five nontrivial eigenvalues of the Hessian $\nabla^{2} W_{\text {cell }}( \pm \widetilde{\mathrm{Id}})$ are given by $V_{1}^{\prime \prime}(1)+2 V_{\sqrt{2}}^{\prime \prime}(\sqrt{2}), V_{1}^{\prime \prime}(1)$, $2 V_{\sqrt{2}}^{\prime \prime}(\sqrt{2})$ and $V_{1}^{\prime \prime}(1)$ (twice). Hence (3.2) is true for $|F \pm \mathrm{Id}| \leq \frac{1}{K}$ if $K$ is big enough. On can see directly that $W_{\text {ref }}(F)$ is strictly positive in the intermediate regime $\frac{1}{K} \leq|F \pm \mathrm{Id}| \leq K$. The same is true for $W_{\text {cell }}$ in the compatible case where $\varepsilon=0$. A standard perturbation argument shows that the strict positivity is not affected by small variations of the potentials $V_{1}$ and $V_{\sqrt{2}}$. By increasing $C$ further if necessary we ensure the validity of (3.2) for all $F$ that are compatible with $y \in \mathcal{A}$.

\subsection{Justification of the limiting theory}

Proposition 3.3. Let the assumptions of Theorem 1.4 be satisfied and $W_{\text {rel }}^{s}(\nu)$ be the surface energy density function defined in (2.13). Then the lower bound

$$
\lim _{\rho \rightarrow \infty} \frac{1}{\rho \varepsilon^{2}}\left(\min _{y \in \mathcal{A}} E^{(\rho)}(\{y\})-W_{\mathrm{cl}}(\mathrm{Id}) \# \mathcal{L}^{(\rho)}-\rho \int_{\partial \Omega} W_{\mathrm{cl}}^{s}(\mathrm{Id}, \nu(s)) \mathrm{d} \mathcal{H}^{1}(s)\right) \geq \sum_{i=1}^{\# \text { edges }} \mathcal{H}^{1}\left(\Gamma_{i}\right) W_{\mathrm{rel}}^{s}\left(\nu_{i}\right)
$$

holds.

Proof. Theorem 1.2 implies that that

$$
\lim _{\rho \rightarrow \infty} \frac{1}{\rho} s_{\mathrm{cl}}^{(\rho)}=\int_{\partial \Omega} W_{\mathrm{cl}}^{s}(\mathrm{Id}, \nu(s)) \mathrm{d} \mathcal{H}^{1}(s)
$$

with

$$
s_{\mathrm{cl}}^{(\rho)}=-\sum_{\substack{x \in \mathcal{L}(\rho) \\ x^{\prime} \in \mathbb{Z}^{2} \backslash \mathcal{L}(\rho)}} V_{\left|x-x^{\prime}\right|}\left(\left|x-x^{\prime}\right|\right)
$$

Thus it suffices to establish

$$
\lim _{\rho \rightarrow \infty} \frac{1}{\rho \varepsilon^{2}}\left(\min _{y \in \mathcal{A}} E^{(\rho)}(\{y\})-W_{\mathrm{cl}}(\mathrm{Id}) \# \mathcal{L}^{(\rho)}-s_{\mathrm{cl}}^{(\rho)}\right) \geq \sum_{i=1}^{\# \text { edges }} \mathcal{H}^{1}\left(\Gamma_{i}\right) W_{\mathrm{rel}}^{s}\left(\nu_{i}\right) .
$$

Throughout the proof we assume that $V_{1}(1)=V_{\sqrt{2}}(\sqrt{2})=0$, this implies of course that $W_{\mathrm{cl}}(\mathrm{Id})=s_{\mathrm{cl}}^{(\rho)}=0$. This simplification does not involve a loss of generality as it can be easily checked that

$$
E^{(\rho)}(\{y\})-W_{\mathrm{cl}}(\mathrm{Id}) \# \mathcal{L}^{(\rho)}-s_{\mathrm{cl}}^{(\rho)}=\sum_{x, x^{\prime} \in \mathcal{L}^{(\rho)}}\left[V_{\mid x-x^{\prime}}\left(\left|y(x)-y\left(x^{\prime}\right)\right|\right)-V_{\left|x-x^{\prime}\right|}\left(\left|x-x^{\prime}\right|\right)\right] .
$$

The proof addresses two separate claims. First we show that the functional form of the limiting energy is correct. Then we justify the periodicity condition which is present in the definition of $W_{\mathrm{rel}}^{s}$.

Step 1. Localize the energy by weighting the pair interaction energy. The main difficulty is to construct domains that localize the surface energy and have bounded rigidity constant. 


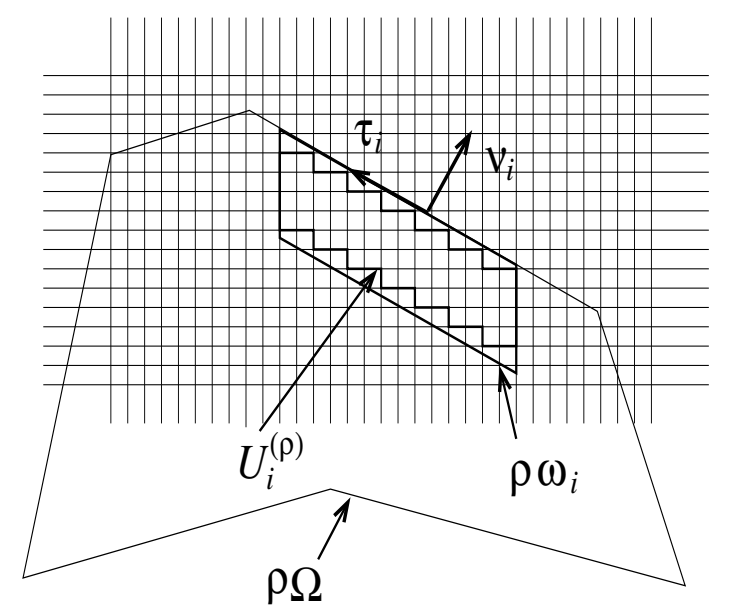

FIGURE 3. The continuum domains $\rho \omega_{i}$ and $U_{i}^{(\rho)}$.

Let $Q=[0,1]^{2}$ be the two-dimensional unit square. A simple calculation shows that there exists a weight distribution $\kappa\left(x, x^{\prime}\right) \in\left\{0, \frac{1}{2}, 1\right\}$ such that

$$
\begin{aligned}
E^{(\rho)}(\{y\})= & \sum_{\substack{x \in \mathbb{Z}^{2} \\
x+Q \subset \rho \Omega}} W_{\text {cell }}\left(y(x), y\left(x+e_{1}\right), y\left(x+e_{1}+e_{2}\right), y\left(x+e_{2}\right)\right) \\
& +\sum_{x, x^{\prime} \in \partial \mathcal{L}^{(\rho)}} \kappa\left(x, x^{\prime}\right) V_{\left|x-x^{\prime}\right|}\left(\left|y(x)-y\left(x^{\prime}\right)\right|\right),
\end{aligned}
$$

and $\kappa\left(x, x^{\prime}\right)=0$ if $\left|x-x^{\prime}\right| \notin\{1, \sqrt{2}\}$. Since $W_{\text {cell }}$ is nonnegative this shows that the relaxation induced surface energy is localized at $\partial \mathcal{L}^{(\rho)}$.

We demonstrate that the energy can be localized within parallelogram sets with small are which cover most of the boundary. Recall that $\partial \Omega$ is a polygon, the $i$-th side is denoted by $\Gamma_{i}$ and $\nu_{i} \in \mathbb{Z}^{2}$ is the associated outward pointing normal vector. Assume without loss of generality that $0 \leq \nu_{1} \leq \nu_{2}$ and let $c_{i, j} \in \mathbb{R}$ be chosen in such a way that

$$
\Gamma_{i}=\left\{\eta \in \mathbb{R}^{2} \mid c_{1, i} \leq \eta \cdot e_{1} \leq c_{2, i} \text { and } \eta \cdot \nu_{i}=c_{3, i}\right\},
$$

where $\tau_{i}$ is the tangent vector or $\Gamma_{i}$. Since the angle between two adjacent edges is strictly bigger than zero there exists a number $\delta \in\left(0, \frac{\pi}{2}\right)$ such that the angle between two neighboring sides is bigger than $\delta$. Hence, for every $\gamma>0$ (which will be sent to 0 later) there exists $c_{4, i}<c_{3, i}$ such that the parallelograms

$$
\omega_{i}=\left\{\eta \in \mathbb{R}^{2} \mid c_{1, i}+\gamma \leq \eta \cdot e_{1} \leq c_{2, i}-\gamma \text { and } c_{3, i} \geq \eta \cdot \nu_{i} \geq c_{4, i}\right\}
$$

are subsets of $\Omega$ and mutually disjoint. Define now the set

$$
U_{i}^{(\rho)}=\cup_{\substack{x \in \mathbb{Z}^{2} \\ x+Q \subset \rho \omega_{i}}}(x+Q),
$$

see Figure 3. The localized energy is given by

$$
E_{i}^{(\rho)}(\{y\}):=\sum_{x \in \mathcal{L}_{i}^{(\rho)}} W_{\text {cell }}\left(y(x), y\left(x+e_{1}\right), y\left(x+e_{1}+e_{2}\right), y\left(x+e_{2}\right)\right)+\sum_{x, x^{\prime} \in \mathcal{I}_{i}^{(\rho)}} \kappa\left(x, x^{\prime}\right) V\left(\left|y(x)-y\left(x^{\prime}\right)\right|\right),
$$


where $\mathcal{L}_{i}^{(\rho)}=\mathcal{L}^{(\rho)} \cap U_{i}^{(\rho)}$ and $\mathcal{I}_{i}^{(\rho)}=\partial \mathcal{L}^{(\rho)} \cap U_{i}^{(\rho)}$. Since $U_{i}^{(\rho)} \subset \rho \omega_{i}$ the sets $U_{i}^{(\rho)}$ are mutually disjoint. A simple Taylor-expansion of the interaction potentials shows that the conditions $V_{\mu}(\mu)=0$ for $\mu \in\{1, \sqrt{2}\}$, (1.3)-(1.6)-(1.9) imply that

$$
\begin{aligned}
& V_{1}(r)=\frac{1}{2} K_{1}\left(r-1+\frac{\varepsilon}{2 K_{1}}\right)^{2}-\frac{\varepsilon^{2}}{8 K_{1}}+o\left(\varepsilon^{2}+(r-1)^{2}\right), \\
& V_{\sqrt{2}}(r)=\frac{1}{2} K_{2}\left(r-\sqrt{2}-\frac{\varepsilon}{2 \sqrt{2} K_{2}}\right)^{2}-\frac{\varepsilon^{2}}{16 K_{2}}+o\left(\varepsilon^{2}+(r-\sqrt{2})^{2}\right)
\end{aligned}
$$

up to an irrelevant ambiguity of the sign in front of the terms of order $\varepsilon$. This representation formula, together with the definition of $E_{i}^{(\rho)}$ and the fact that the sets $U_{i}^{(\rho)}$ are disjoint, implies the localization bound

$$
E^{(\rho)}(\{y\}) \geq \sum_{i=1}^{\# \text { edges }} E_{i}^{(\rho)}(\{y\})-C \varepsilon^{2} \rho \gamma .
$$

Note that the restriction $y \in \mathcal{A}$ enables us to rewrite $E^{(\rho)}$ in terms of $W_{\text {cell }}$, without the determinant constraint the estimate does not hold. As $\gamma>0$ can be made arbitrarily small, this shows that we are done once we are able to prove that

$$
\liminf _{\rho \rightarrow \infty} \inf _{y} E_{i}^{(\rho)}(\{y\}) \geq\left(\mathcal{H}^{1}\left(\Gamma_{i}\right)-2 \gamma\right) W_{\text {rel }}^{s}\left(\nu_{i}\right) .
$$

To simplify the notation we choose weights $w_{i}\left(x, x^{\prime}\right) \in\left\{0, \frac{1}{2}, 1\right\}$ such that

$$
E_{i}^{(\rho)}(\{y\})=\sum_{x, x^{\prime} \in \mathcal{L}_{i}^{(\rho)}} w_{i}\left(x, x^{\prime}\right) V\left(\left|y(x)-y\left(x^{\prime}\right)\right|\right) .
$$

Step 2. Obtain a discrete rigidity estimate.

During the following considerations the index $i$ is fixed and will not be shown in most cases.

Lemma 3.4. The rigidity constant of the set $U_{i}^{(\rho)}$ which is defined in Theorem 3.1 remains bounded as $\rho$ tends to infinity.

Proof. First note that the rigidity constant is unchanged by translations, rotations and dilations.

Since the index $i$ is fixed it will not be shown within this proof. The rigidity constant of $U^{(\rho)}$ can be estimated by a combination of two different Poincaré constants which remain bounded under bi-Lipschitz transformations of the domain. We construct a bi-Lipschitz map $\phi^{(\rho)}: \omega \rightarrow \frac{1}{\rho} U^{(\rho)}$ with bounded Lipschitz constants. The set $U^{(\rho)}$ is fibrated by line segments which are parallel to $\nu$, and $\phi^{(\rho)}$ is an affine stretch along each line segment.

Define for each $\rho$ the affine function $\xi$ which satisfies

$$
\xi(\min \{\eta \cdot \tau \mid \eta \in \omega\})=\min \left\{\frac{1}{\rho} \eta \cdot \tau \mid \eta \in U^{(\rho)}\right\},
$$

and an analogous condition involving the corresponding maxima. Clearly $\xi$ converges to the identity as $\rho$ tends to infinity.

Define next the maximum heights

$$
\begin{aligned}
& \lambda_{+}(s)=\max \{\eta \cdot \nu \mid \eta \in \omega \text { and } \eta \cdot \tau=s\}, \\
& \psi_{+}(s)=\max \left\{\eta \cdot \nu \mid \eta \in \frac{1}{\rho} U^{(\rho)} \text { and } \eta \cdot \tau=\xi(s)\right\},
\end{aligned}
$$

and denote the corresponding minimum heights by $\psi_{-}, \lambda_{-}$. 
Using the definition of the sets $U^{(\rho)}$ and $\omega$ it is a simple exercise to check that there exists a constant $C$ which depends on $\nu$, but not on $\rho$ such that:

(1) $\lambda_{ \pm}$and $\psi_{ \pm}$are Lipschitz-continuous with Lipschitz constants bounded by $C$;

(2) The bound

$$
\frac{1}{C}\left(\lambda_{+}-\lambda_{-}\right) \leq \psi_{+}-\psi_{-} \leq C\left(\lambda_{+}-\lambda_{-}\right)
$$

is satisfied.

The map $\phi: \rho \omega \rightarrow U^{(\rho)}$ is defined by

$$
\phi(\eta)=\frac{1}{|\nu|^{2}}\left(\psi_{-}+\frac{\psi_{+}-\psi_{-}}{\lambda_{+}-\lambda_{-}}\left(\eta \cdot \nu-\lambda_{-}\right)\right) \nu+\frac{1}{|\tau|^{2}} \xi \tau
$$

with the convention that the argument of the function $\xi, \lambda, \psi$ is $\tau \cdot \eta$. The gradient of $\phi^{(\rho)}$ is given by

$$
\begin{aligned}
\nabla \phi \nu= & \frac{\psi_{+}-\psi_{-}}{\lambda_{+}-\lambda_{-}} \nu \\
\nabla \phi \tau= & \frac{|\tau|^{2}}{|\nu|^{2}}\left(\psi_{-}^{\prime}-\frac{\psi_{+}-\psi_{-}}{\lambda_{+}-\lambda_{-}} \lambda_{-}^{\prime}+\frac{\eta \cdot \nu-\lambda_{-}}{\lambda_{+}-\lambda_{-}}\left(\psi_{+}^{\prime}-\psi_{-}^{\prime}\right)\right. \\
& \left.-\frac{\left(\eta \cdot \nu-\lambda_{-}\right)\left(\psi_{+}-\psi_{-}\right)}{\left(\lambda_{+}-\lambda_{-}\right)^{2}}\left(\lambda_{+}^{\prime}-\lambda_{-}^{\prime}\right)\right) \nu+\xi^{\prime} \tau
\end{aligned}
$$

The Lipschitz continuity of $\psi_{ \pm}$and $\lambda_{ \pm}$together with the bound (3.10) implies that each term is bounded. The inverse of $\phi^{(\rho)}$ can be estimated in an analogous fashion.

Let $y_{i}^{(\rho)}$ be a minimizer of the lattice energy $E_{i}^{(\rho)}(\cdot)$. Formula (3.6) implies that

$$
E_{i}^{(\rho)}\left(\left\{y_{i}^{(\rho)}\right\}\right) \geq \sum_{\substack{x \in \mathbb{Z}^{2} \\ x+Q \subset U_{i}^{(\rho)}}} W_{\text {cell }}\left(y^{(\rho)}(x), y^{(\rho)}\left(x+e_{1}\right), y^{(\rho)}\left(x+e_{1}+e_{2}\right), y^{(\rho)}\left(x+e_{2}\right)\right)-C \varepsilon^{2} \rho .
$$

Let $u^{(\rho)} \in W^{1, \infty}\left(U^{(\rho)}\right)$ such that:

(1) $u^{(\rho)}$ is affine within the sets $\operatorname{conv}\left\{x, x+e_{1}, x+e_{2}\right\} \cap U^{(\rho)}, \operatorname{conv}\left\{x, x-e_{1}, x-e_{2}\right\} \cap U^{(\rho)}$ for each $x \in \mathbb{Z}^{2}$;

(2) $u^{(\rho)}$ interpolates between the values of $y^{(\rho)}$.

Since the trial-map $y(x)=x$ is admissible we obtain the bound

$$
E_{i}^{(\rho)}\left(\left\{y^{(\rho)}\right\}\right) \leq 0
$$

Use (3.11) and (3.2) to deduce that

$$
\int_{U_{i}^{(\rho)}}\left|\nabla u^{(\rho)}-S O(2)\right|^{2} \mathrm{~d} \eta \leq C \varepsilon^{2} \rho
$$

Next, we apply the rigidity estimate (3.1) and obtain the existence of a rotation matrix $R \in S O(2)$ such that

$$
\int_{U(\rho)}\left|\nabla u^{(\rho)}-R\right|^{2} \mathrm{~d} \eta \leq C \varepsilon^{2} \rho .
$$


Simple algebraic manipulations show that

$$
\sum_{\substack{x^{\prime}, x^{\prime \prime} \in(x+Q) \cap \mathbb{Z}^{2} \\\left|x^{\prime}-x^{\prime \prime}\right|=1}}\left|y^{(\rho)}\left(x^{\prime}\right)-y^{(\rho)}\left(x^{\prime \prime}\right)-R\left(x^{\prime}-x^{\prime \prime}\right)\right|^{2}=4 \int_{x+Q}\left|\nabla u^{(\rho)}-R\right|^{2} \mathrm{~d} \eta
$$

and together with the triangle inequality one finds that

$$
\sum_{x, x^{\prime} \in \mathcal{L}^{(\rho)}} w_{i}\left(x, x^{\prime}\right)\left|y^{(\rho)}(x)-y^{(\rho)}\left(x^{\prime}\right)-R\left(x-x^{\prime}\right)\right|^{2} \leq C \rho \varepsilon^{2} .
$$

To simplify the notation we define $z^{(\rho)}(x)=\frac{1}{\varepsilon}\left(y^{(\rho)}(x)-R x\right)$ and obtain the a priori estimate

$$
\sum_{x, x^{\prime} \in \mathcal{L}^{(\rho)}} w\left(x, x^{\prime}\right)\left|z^{(\rho)}(x)-z^{(\rho)}\left(x^{\prime}\right)\right|^{2} \leq C \rho .
$$

In particular, this delivers the bound

$$
\max _{x, x^{\prime}} w\left(x, x^{\prime}\right)\left|z^{(\rho)}(x)-z^{(\rho)}\left(x^{\prime}\right)\right| \leq C \sqrt{\rho} .
$$

Step 3. Remove those terms where $\left|y^{(\rho)}(x)-y^{(\rho)}\left(x^{\prime}\right)-x+x^{\prime}\right|$ is not small.

Using the representation formula (3.7) we obtain that

$$
V_{\left|x-x^{\prime}\right|}\left(\left|y(x)-y\left(x^{\prime}\right)\right|\right)=\varepsilon^{2}\left(\frac{1}{2} A\left(z(x)-z\left(x^{\prime}\right)\right)+L\right) \cdot\left(z(x)-z\left(x^{\prime}\right)\right)+h\left(\varepsilon\left(z(x)-z\left(x^{\prime}\right)\right)\right),
$$

where $h=h_{x-x^{\prime}},|h(G)|=o\left(|G|^{2}\right)$ as $G \rightarrow 0$ and $L$ and $A$ are defined by equations (2.1) and (2.2). Let

$$
\chi\left(x, x^{\prime}\right):= \begin{cases}1, & \text { if }\left|z^{(\rho)}(x)-z^{(\rho)}\left(x^{\prime}\right)\right| \leq \varepsilon^{-\frac{1}{2}} \text { and }\left|x-x^{\prime}\right| \leq \sqrt{2} \\ 0, & \text { else }\end{cases}
$$

be the indicator function of the good bonds. The a priori estimate (3.13) implies that

$$
\sum_{x, x^{\prime} \in \mathcal{L}^{(\rho)}} w\left(x, x^{\prime}\right)\left(1-\chi\left(x, x^{\prime}\right)\right) \leq C \rho \varepsilon
$$

In particular, the number of "bad" bonds is $o(\rho)$ as $\rho \rightarrow \infty$. This estimate can be used to show that neglecting energy concentrations does not affect the asymptotic behavior of the surface energy:

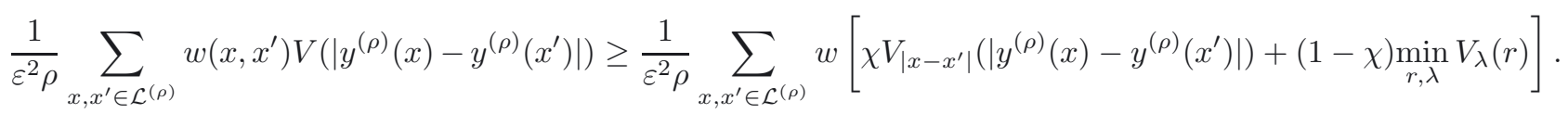

As $\min _{r, \lambda} V_{\lambda}(r)=O\left(\varepsilon^{2}\right)$ as $\varepsilon \rightarrow 0$, together with (3.15) this proves that the last term vanishes as $\rho \rightarrow \infty$. 
Now we are in the position to exploit the differentiability of the potentials in the following sense

$$
\begin{aligned}
\frac{1}{\rho \varepsilon^{2}} \sum_{x, x^{\prime} \in \mathcal{L}^{(\rho)}} w \chi V\left(\left|\left(x-x^{\prime}\right)+\varepsilon\left(z^{(\rho)}(x)-z^{(\rho)}\left(x^{\prime}\right)\right)\right|\right)= & \frac{1}{\rho} \sum_{x, x^{\prime} \in \mathcal{L}^{(\rho)}} w\left[\frac { 1 } { 2 } \chi ( z ^ { ( \rho ) } ( x ) - z ^ { ( \rho ) } ( x ^ { \prime } ) ) \cdot A \chi \left(z^{(\rho)}(x)\right.\right. \\
& \left.\left.-z^{(\rho)}\left(x^{\prime}\right)\right)+\frac{\chi}{\varepsilon^{2}} h\left(\varepsilon\left(z^{(\rho)}(x)-z^{(\rho)}\left(x^{\prime}\right)\right)\right)\right] \\
& +\sum_{x, x^{\prime} \in \partial \mathcal{L}(\rho)} w L \cdot \chi\left(z^{(\rho)}(x)-z^{(\rho)}\left(x^{\prime}\right)\right) .
\end{aligned}
$$

We rewrite the error term in (3.16) slightly and obtain that

$\lim _{\rho \rightarrow \infty} \frac{1}{\varepsilon^{2} \rho} \sum_{x, x^{\prime} \in \mathcal{L}^{(\rho)}} \chi w h\left(\varepsilon\left(z^{(\rho)}(x)-z^{(\rho)}\left(x^{\prime}\right)\right)\right)=\lim _{\rho \rightarrow \infty} \sum_{x, x^{\prime} \in \mathcal{L}^{(\rho)}} \chi w \frac{1}{\rho}\left|z^{(\rho)}(x)-z^{(\rho)}\left(x^{\prime}\right)\right|^{2} \frac{h\left(\varepsilon\left(z^{(\rho)}(x)-z^{(\rho)}\left(x^{\prime}\right)\right)\right)}{\left(\varepsilon\left|z^{(\rho)}(x)-z^{(\rho)}\left(x^{\prime}\right)\right|\right)^{2}}=0$

since the last term is the product of a bounded sequence in $l^{1}$ and a sequence which converges to 0 in $l^{\infty}$ as $\rho \rightarrow \infty$. Hence, we have demonstrated that

$$
\begin{aligned}
& \liminf _{\rho \rightarrow \infty} \frac{1}{\rho \varepsilon^{2}} \sum_{x, x^{\prime} \in \mathcal{L}^{(\rho)}} w \chi V\left(\left|\left(x-x^{\prime}\right)+\varepsilon\left(z^{(\rho)}(x)-z^{(\rho)}\left(x^{\prime}\right)\right)\right|\right) \\
& =\liminf _{\rho \rightarrow \infty} \frac{1}{2 \rho} \sum_{x, x^{\prime} \in \mathcal{L}^{(\rho)}} w \chi\left(z^{(\rho)}(x)-z^{(\rho)}\left(x^{\prime}\right)\right) \cdot A \chi\left(z^{(\rho)}(x)-z^{(\rho)}\left(x^{\prime}\right)\right) \\
& +\liminf _{\rho \rightarrow \infty} \sum_{x, x^{\prime} \in \mathcal{I}(\rho)} \chi w L \cdot\left(z^{(\rho)}(x)-z^{(\rho)}\left(x^{\prime}\right)\right) .
\end{aligned}
$$

Step 4. Demonstrate that the weak limit of the averaged differences is a gradient.

It remains to be shown that the right hand side in $(3.16)$ is bounded from below by $\frac{1}{\left|\tau_{i}\right|}\left(\mathcal{H}^{1}\left(\Gamma_{i}\right)-2 \gamma\right) \min _{z \in \mathcal{A}_{\nu_{i}}}$ $Q_{\nu_{i}}(\{z\})$.

We use a specifically adapted notion of weak convergence where averaging is only performed in the tangential direction, not in the normal direction.

Let

$$
K_{i}=\rho \frac{\mathcal{H}^{1}\left(\Gamma_{i}\right)-2 \gamma}{\left|\tau_{i}\right|}
$$

and $x_{i}^{(\rho)} \in \mathcal{I}_{i}^{(\rho)}$ be extremal in the sense that $\left(x_{i}^{(\rho)}-x\right) \cdot \nu_{i} \geq 0$ for all $x \in U_{i}^{(\rho)} \cap \mathbb{Z}^{2}$. For each pair $x, x^{\prime} \in \mathbb{Z}_{\nu}^{2}$ the averaged differences are defined by

$$
g^{(\rho)}\left(x, x^{\prime}\right):=\frac{1}{K_{i}} \sum_{l \in Z\left(x, x^{\prime}\right)}\left(z^{(\rho)}\left(x^{(\rho)}+x+l \tau\right)-z^{(\rho)}\left(x^{(\rho)}+x^{\prime}+l \tau\right)\right),
$$

where

$$
Z_{i}\left(x, x^{\prime}\right)=\left\{l \in \mathbb{Z} \mid x^{(\rho)}+l \tau+\left\{x, x^{\prime}\right\} \subset U^{(\rho)}\right\}
$$

Using the definition of $U^{(\rho)}$ one can easily deduce that

$$
\lim _{\rho \rightarrow \infty} \frac{\# Z_{i}\left(x, x^{\prime}\right)}{K_{i}}=1
$$


We will demonstrate now that there exists a $\tau$-periodic map $\zeta: \mathbb{Z}_{\nu}^{2} \rightarrow \mathbb{R}^{2}$ and a subsequence of $g^{(\rho)}$ such that

$$
g\left(x, x^{\prime}\right):=\lim _{\rho \rightarrow \infty} g^{(\rho)}\left(x, x^{\prime}\right)=\zeta(x)-\zeta\left(x^{\prime}\right) \text { for all } x, x^{\prime} \in \mathbb{Z}_{\nu}^{2}
$$

First we will show that $g$ can be written as differences of some map $\zeta$. In a second step the $\tau$-periodicity of $\zeta$ will be established.

Equation (3.19) holds if we can show that for all closed paths $\left(x_{r}\right)_{r=0 \ldots R} \in \mathbb{Z}_{\nu}^{2}$ the equation

$$
\sum_{r=1}^{R} g\left(x_{r}, x_{r-1}\right)=0
$$

holds true. Indeed, thanks to the definition of $U^{(\rho)}$ and since the diameter of the path is finite, for each $r$ the set $Z\left(x_{r}, x_{r-1}\right)$ can be partitioned into two sets $Z^{g}, Z^{b} \subset Z\left(x_{r}, x_{r-1}\right)$ such that the set $Z^{g}$ does not depend on $r$ and $\# Z^{b}$ is bounded independently of $\rho$ and $r$. With this notation one finds

$$
\begin{aligned}
& \left|\sum_{r=1}^{R} g^{(\rho)}\left(x_{r}, x_{r-1}\right)\right| \leq\left|\sum_{r=1}^{R} \frac{1}{K} \sum_{l \in Z^{g}}\left(z^{(\rho)}\left(x^{(\rho)}+x_{r}+l \tau\right)-z^{(\rho)}\left(x^{(\rho)}+x_{r-1}+l \tau\right)\right)\right| \\
& +\left|\sum_{r=1}^{R} \frac{1}{K} \sum_{l \in Z^{b}}\left(z^{(\rho)}\left(x^{(\rho)}+x_{r}+l \tau\right)-z^{(\rho)}\left(x^{(\rho)}+x_{r-1}+l \tau\right)\right)\right| .
\end{aligned}
$$

Swapping the sums in the first term shows that it is identically zero since the path is closed, i.e. $x_{0}=x_{R}$. Thanks to (3.14) the second term is bounded by $C \frac{\# Z^{b} \sqrt{\rho}}{K_{i}}$, which converges to 0 as $\rho$ tends to infinity. This shows that there is a subsequence and a map $\zeta$ such that equation (3.19) holds.

It remains to show that $\zeta$ is $\tau$-periodic. To see this we define the defect

$$
\delta(x)=\sum_{r=1}^{R} g\left(x_{r}, x_{r-1}\right)
$$

for some path $\left(x_{r}\right)_{r=0 \ldots R}$ such that $x_{0}=x$ and $x_{R}=x+\tau$. We will show that $\delta(x)=\delta\left(x^{\prime}\right)$ for all $x, x^{\prime} \in \mathbb{Z}_{\nu}^{2}$. Let $\left(x_{k}^{\prime}\right)_{k=0 \ldots K}$ be a closed path that visits $x, x+\tau, x^{\prime}+\tau, x^{\prime}$ (in that order). Repeating the argument above we find that $\delta(x)=\delta\left(x^{\prime}\right)$.

If $\delta>0$, then one obtains an contradiction to (3.13) since every $x \in \mathbb{Z}_{\nu}^{2} / \tau$ (the semi-infinite cylinder where translatations by $\tau$ are identified) generates a positive contribution of order $\rho$. Therefore, $\delta=0$ which implies that for any $x^{\prime} \in \mathbb{Z}_{\nu}^{2}$ the map $\zeta(x)=g\left(x, x^{\prime}\right)$ is $\tau$-periodic in $x$.

Armed with these results we are in the position to establish the following asymptotic result for the linear term in (3.16). Let $x, x^{\prime} \in \mathbb{Z}_{\nu}^{2}$ and define

$$
J_{1}^{(\rho)}\left(x, x^{\prime}\right)=\frac{1}{\rho} \sum_{l \in Z\left(x, x^{\prime}\right)} w \chi L \cdot\left(z^{(\rho)}\left(x^{(\rho)}+x+l \tau\right)-z^{(\rho)}\left(x^{(\rho)}+x^{\prime}+l \tau\right)\right) .
$$

We isolate the bad terms

$$
\begin{aligned}
\lim _{\rho \rightarrow \infty} J_{1}^{(\rho)}= & \lim _{\rho \rightarrow \infty} \frac{1}{\rho} \sum_{l \in Z\left(x, x^{\prime}\right)} w L \cdot\left(z^{(\rho)}\left(x^{(\rho)}+x+l \tau\right)-z^{(\rho)}\left(x^{(\rho)}+x^{\prime}+l \tau\right)\right) \\
& -\lim _{\rho \rightarrow \infty} \frac{1}{\rho} \sum_{l \in Z\left(x, x^{\prime}\right)} w(1-\chi) L \cdot\left(z^{(\rho)}\left(x^{(\rho)}+x+l \tau\right)-z^{(\rho)}\left(x^{(\rho)}+x^{\prime}+l \tau\right)\right),
\end{aligned}
$$


and apply equation (3.15) which implies that the second limit is zero. Like before it is possible to partition $Z$ into $Z^{g}$ and $Z^{b}$ such that $\# Z^{b}$ remains bounded as $\rho$ tends to infinity and $w\left(x^{(\rho)}+x+l \tau, x^{(\rho)}+x^{\prime}+l \tau\right)=v_{\nu}\left(x, x^{\prime}\right)$ for all $l \in Z^{g}$, where $v_{\nu}$ is defined in (2.5). Hence

$$
\lim _{\rho \rightarrow \infty} J_{1}^{(\rho)}=v_{\nu}\left(x, x^{\prime}\right) L\left(x-x^{\prime}\right) \cdot \lim _{\rho \rightarrow \infty} \frac{1}{\rho} \sum_{l \in Z^{g}}\left(z^{(\rho)}\left(x^{(\rho)}+x+l \tau\right)-\zeta\left(x^{(\rho)}+x^{\prime}+l \tau\right)\right) .
$$

Next we apply the definition of $K$ (Eq. (3.17)) and the convergence (3.19) and find that

$$
\begin{aligned}
\lim _{\rho \rightarrow \infty} J_{1}^{(\rho)}\left(x, x^{\prime}\right) & =\frac{\mathcal{H}^{1}(\Gamma)-2 \gamma}{|\tau|} v_{\nu}\left(x, x^{\prime}\right) L\left(x-x^{\prime}\right) \lim _{\rho \rightarrow \infty} \frac{1}{K} \sum_{l \in Z\left(x, x^{\prime}\right)}\left(z^{(\rho)}\left(x^{(\rho)}+x+l \tau\right)-z^{(\rho)}\left(x^{(\rho)}+x^{\prime}+l \tau\right)\right) \\
& =\frac{\mathcal{H}^{1}(\Gamma)-2 \gamma}{|\tau|} v_{\nu}\left(x, x^{\prime}\right) L\left(x-x^{\prime}\right) \cdot\left(\zeta(x)-\zeta\left(x^{\prime}\right)\right) .
\end{aligned}
$$

The definition of the boundary forces $b_{\nu}$ (Eq. (2.4)) and the definition of the weights $v_{\nu}$ (analogous to $w$ ) delivers the final form of the linear terms

$$
\lim _{\rho \rightarrow \infty} \frac{1}{2} \sum_{x, x^{\prime} \in \mathbb{Z}_{\nu}^{2}} J_{1}^{(\rho)}\left(x, x^{\prime}\right)=\frac{\mathcal{H}^{1}(\Gamma)-2 \gamma}{|\tau|} \sum_{x \in \partial \mathbb{Z}_{\nu}^{2} \bmod \tau} \zeta(x) \cdot b_{\nu}(x)
$$

The limit of the quadratic term in (3.16) can found in a similar fashion. Let $x, x^{\prime} \in \mathbb{Z}_{\nu}^{2}$ and define

$J_{2}^{(\rho)}\left(x, x^{\prime}\right)=\frac{1}{\rho} \sum_{l \in \mathbb{Z}} w \chi\left(z^{(\rho)}\left(x^{(\rho)}+x+l \tau\right)-z^{(\rho)}\left(x^{(\rho)}+x^{\prime}+l \tau\right)\right) \cdot A\left(z^{(\rho)}\left(x^{(\rho)}+x+l \tau\right)-z^{(\rho)}\left(x^{(\rho)}+x^{\prime}+l \tau\right)\right)$.

The convexity of the matrix $A$ together with the definition of $K$ (Eq. (3.17)) implies for each $x, x^{\prime} \in \mathbb{Z}_{\nu}^{2}$

$$
\liminf _{\rho \rightarrow \infty} J_{2}^{(\rho)} \geq \frac{\mathcal{H}^{1}(\Gamma)-2 \gamma}{|\tau|} \liminf _{\rho \rightarrow \infty} g_{1}^{(\rho)}\left(x, x^{\prime}\right) \cdot A g_{1}^{(\rho)}\left(x, x^{\prime}\right)
$$

where $g_{1}^{(\rho)}=\frac{1}{K} \sum_{l \in Z\left(x, x^{\prime}\right)} w \chi\left(z^{(\rho)}\left(x^{(\rho)}+x+l \tau\right)-z^{(\rho)}\left(x^{(\rho)}+x^{\prime}+l \tau\right)\right)$. Estimate (3.15) implies that $\lim _{\rho \rightarrow \infty} g_{1}^{(\rho)}\left(x, x^{\prime}\right)=g\left(x, x^{\prime}\right)$ and thus

$$
\lim _{\rho \rightarrow \infty} J_{2}^{(\rho)}=\frac{\mathcal{H}^{2}(\Gamma)-2 \gamma}{|\tau|}\left(\zeta(x)-\zeta\left(x^{\prime}\right)\right) \cdot A\left(\zeta(x)-\zeta\left(x^{\prime}\right)\right) .
$$

Equations (3.16), (3.21) and (3.22) together imply the estimate

$$
\liminf _{\rho \rightarrow \infty} \frac{1}{\rho \varepsilon^{2}} E_{i}^{(\rho)}\left(\left\{y^{(\rho)}\right\}\right) \geq \frac{\mathcal{H}^{1}(\Gamma)-2 \gamma}{|\tau|} \inf _{z} Q_{\nu_{i}}(\{z\})=\left(\mathcal{H}^{1}\left(\Gamma_{i}\right)-2 \gamma\right) W_{\text {rel }}^{s}\left(\nu_{i}\right)
$$

and we have established that (3.8) indeed holds.

Proof of Theorem 1.4. All we have to do is construct a lattice deformation that exhausts the lower bound (3.5). As before we assume that without loss of generality $V_{1}(1)=V_{\sqrt{2}}(\sqrt{2})=0$. For $i \in\{1, \ldots$,\# \#dges $\}$ let $U_{i}^{(\rho)} \subset \mathbb{R}^{2}$ be the rectangle that has been defined in the proof of Proposition 3.3 and recall the definition of $x_{i}^{(\rho)}$ from Step 4 . We define the trial field

$$
z_{\text {trial }}^{(\rho)}(x)=\left\{\begin{aligned}
z_{\nu_{i}}\left(x-x_{i}^{(\rho)}\right)-z_{\nu_{i}}^{\infty}, & \text { if } x \in U_{i}^{(\rho)} \cap \mathbb{Z}^{2}, \\
0, & \text { else }
\end{aligned}\right.
$$


where $z_{\nu}$ and $z_{\nu}^{\infty}$ are given in Theorem 2.1. Set $y_{\text {trial }}^{(\rho)}(x)=x+\varepsilon z_{\text {trial }}^{(\rho)}(x)$. The exponential decay estimate (2.11) implies that $\left|y_{\text {trial }}^{(\rho)}(x)-x\right| \leq C \varepsilon \exp \left(-\frac{1}{C} \operatorname{dist}\left(x, \partial \mathcal{L}^{(\rho)}\right)\right)$ holds. For sufficiently large $\rho$ one obtains that $C \varepsilon \leq \frac{1}{2 \sqrt{2}}$, and consequentially $y_{\text {trial }}^{(\rho)}$ is admissible.

Hence, we obtain that

$$
\begin{aligned}
\lim _{\rho \rightarrow \infty} \frac{1}{\varepsilon^{2} \rho} E^{(\rho)}\left(\left\{y_{\text {trial }}^{(\rho)}\right\}\right)= & \lim _{\rho \rightarrow \infty} \sum_{i=1}^{\# \text { edges }} \frac{1}{\left|\tau_{i}\right|}\left(\mathcal{H}^{1}(\Gamma)-2 \gamma\right) \sum_{\left(x, x^{\prime}\right) \in \mathcal{B}_{\nu_{i}}}\left[\left(\frac{1}{2} A\left(z_{\nu_{i}}(x)-z_{\nu_{i}}\left(x^{\prime}\right)\right)+L\right)\right. \\
& \left.\cdot\left(z_{\nu_{i}}(x)-z_{\nu_{i}}\left(x^{\prime}\right)\right)+\frac{1}{\varepsilon^{2}} h\left(\varepsilon\left(z_{\nu_{i}}(x)-z_{\nu_{i}}\left(x^{\prime}\right)\right)\right)\right] \\
= & \sum_{i=1}^{\# \text { edges }}\left(\mathcal{H}^{1}\left(\Gamma_{i}\right)-2 \gamma\right) Q_{\nu_{i}}\left(\left\{z_{\nu_{i}}\right\}\right)=\sum_{i=1}^{\# \text { edges }}\left(\mathcal{H}^{1}\left(\Gamma_{i}\right)-2 \gamma\right) W_{\text {rel }}^{(s)}\left(\nu_{i}\right)
\end{aligned}
$$

by definition of $W_{\text {rel }}^{(s)}$. As $\gamma$ can be made arbitrarily small the upper bound agrees with the lower bound.

\section{VisuALIZATION OF THE SURFACE ENERGY DENSITY FUNCTIONS}

Due to the short-range character of the potentials the clamped surface energy density function (see Def. 1.1) can be evaluated explicitly:

$$
W_{\mathrm{cl}}^{s}(\nu)=\frac{1}{|\nu|_{2}}\left(|\nu|_{1} V_{1}(1)+2|\nu|_{\infty} V_{\sqrt{2}}(\sqrt{2})\right) .
$$

Obviously $W_{\mathrm{cl}}^{s}(\nu)$ depends in a smooth way on $\nu$ except on the set $\left.\left\{\nu \in \mathbb{R}^{2} \mid \nu_{1} \nu_{2}\left(\nu_{1}+\nu_{2}\right)\left(\nu_{1}-\nu_{2}\right)=0\right\}\right)$. The total surface energy is now defined as

$$
W_{\varepsilon}^{s}(\nu)=W_{\mathrm{cl}}^{s}(\nu)+\varepsilon^{2} W_{\mathrm{rel}}^{s}(\nu) .
$$

It is customary to visualize the surface energy via the Frank diagram. To this end we define $f_{\varepsilon}$ to be the homogeneous of degree one extension of $W_{\varepsilon}^{s}\left(\frac{1}{|\nu|} \nu\right)$ in the sense that

$$
f_{\varepsilon}(\nu)=W_{\varepsilon}^{s}\left(\frac{1}{|\nu|} \nu\right) \nu .
$$

The Frank diagram is the level set of $\left\{f_{\varepsilon}(\nu)=1\right\}$. The significance of this construction is given by the observation that the Frank diagram bounds a convex set if and only if all possible surfaces are stable, i.e. replacing a particular surface by a mixture of different surfaces with the same average normal vector does not lower the total energy.

The Frank diagrams for $W_{\varepsilon}^{s}$ and $W_{\mathrm{cl}}^{s}$ can be compared in Figure 4. The numerical results suggest that $\nu \mapsto W_{\varepsilon}^{s}(\nu)$ is nowhere smooth due to the relaxation effects, $c f$. the plot of the difference quotients in Figure 2. A typical relaxation pattern can be seen in Figure 5.

Conjecture. The function $\nu \mapsto W_{\text {rel }}^{s}(\nu)$ is not continuously differentiable on the set $\mathbb{Q}^{2} \backslash\{0\}$, see Figure 2 .

\section{Possible extensions}

(1) It would be interesting to obtain an asymptotic expression for the surface energy-density in the spirit of homogenization theory for bulk models ( $c f$. [5]) without linearizing the system, i.e. assume $\varepsilon=O(1)$ instead of $\varepsilon=o(1)$ in Theorem 1.4. 


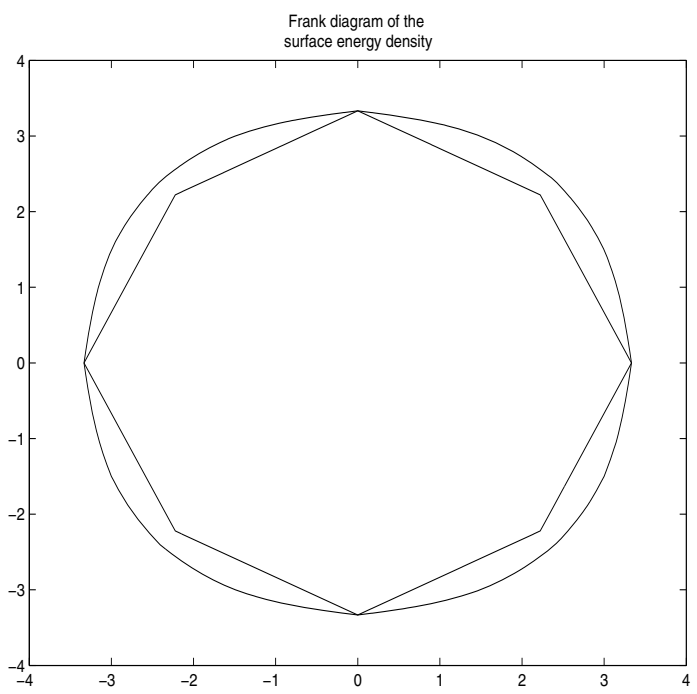

Figure 4. The Frank diagram of the surface energy without relaxation effects (inner line) and with relaxation effects (outer line) for $\varepsilon=0.1, K_{1}=K_{2}=1$.

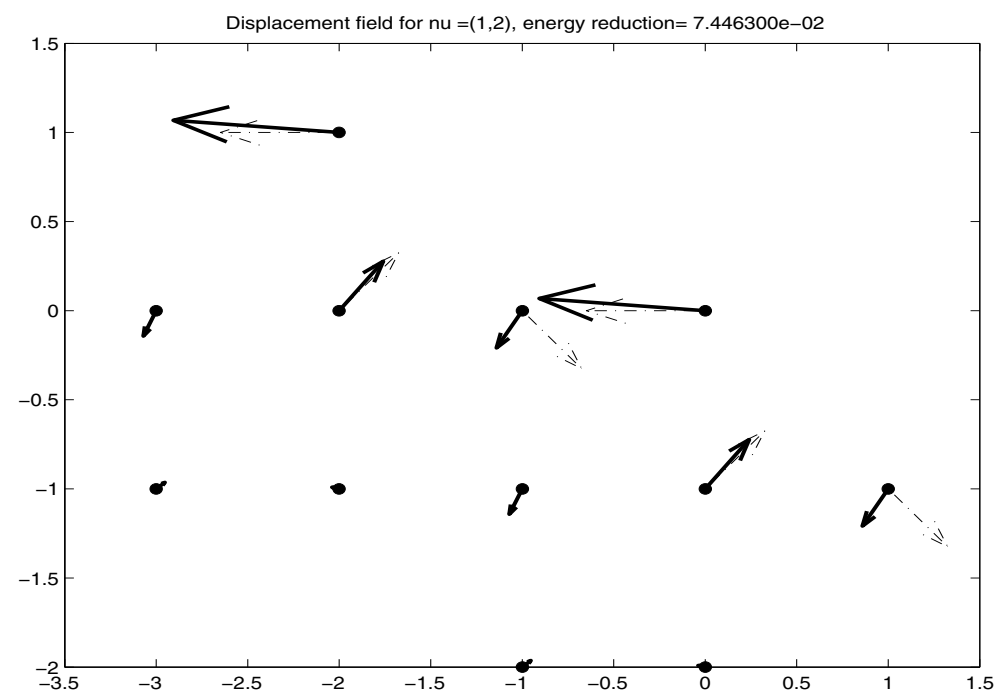

FiguRE 5 . The relaxation pattern for $\nu=(1,2), K_{1}=K_{2}=1$. The solid arrows show the displacements, the dashed arrows illustrate the forcing due to missing bonds.

(2) The model could also be used in order to describe crystal growth. The simplest approach would be to seek the asymptotic shape of

$$
\left\{\frac{1}{\sqrt{\rho}} y^{(\rho)}(x) \mid x \in \mathcal{L}^{(\rho)}\right\}
$$

as $\rho \rightarrow \infty$, where the pair $\left(y^{(\rho)}, \mathcal{L}^{(\rho)}\right)$ is found by minimizing the energy $E^{(\mathcal{L})}(\{y\})$ subject to the constrain that $\mathcal{L} \subset \mathbb{Z}^{2}$ and $\# \mathcal{L} \leq \rho$.

Conjecture. Let $\sigma$ be the surface energy density that is obtained by replacing the quadratic potentials in (2.7) with the original potentials $V_{1}$ and $V_{\sqrt{2}}$. If $|\varepsilon|$ is sufficiently small, here exists a number 
$\lambda \in(0, \infty)$, a translation $c_{\rho} \in \mathbb{R}^{2}$ and a rotation $R_{\rho} \in S O(2)$ such that

$$
\lim _{\rho \rightarrow \infty} R_{\rho}\left\{\frac{1}{\sqrt{\rho}} y^{(\rho)}(x)-c_{\rho} \mid x \in \mathcal{L}^{(\rho)}\right\}=\bigcap_{\nu \in \mathbb{R} \mathbb{Z}^{2} \backslash\{0\}}\{(x \cdot \nu) \leq \lambda \sigma(\nu)|\nu|\} .
$$

The set on the right hand side of the last equation is also known under the name "Wulff shape".

\section{APPENDiX}

Proof of Proposition 1.5. A) Let $y^{(\rho)}$ be the minimizer of $E^{(\rho)}(\{\cdot\})$. After changing the order of summation we obtain the representation

$$
\begin{aligned}
\sigma_{\text {end }}^{(\rho)}= & \frac{1}{2}\left(V_{1}\left(y^{(\rho)}(1)-y^{(\rho)}(0)\right)+V_{1}\left(y^{(\rho)}(\rho)-y^{(\rho)}(\rho-1)\right)\right)-2 W_{\mathrm{cl}}(\mathrm{Id}) \\
& +\sum_{x=1}^{\rho-1} W_{\text {cell }}\left(y^{(\rho)}(x-1), y^{(\rho)}(x), y^{(\rho)}(x+1)\right),
\end{aligned}
$$

where $W_{\text {cell }}(a, b, c)=\frac{1}{2}\left(V_{1}(b-a)+V_{1}(c-b)\right)+V_{2}(c-a)-W_{\mathrm{cl}}(\mathrm{Id})$. The convexity of $V_{1}$ and $V_{2}$ together with (1.10) implies that $W_{\text {cell }}(a, b, c) \geq W_{\text {cell }}(-1,0,1)=0$ for all $a \leq b \leq c$, this gives the lower bound $\sigma_{\text {end }}^{(\rho)} \geq \min V_{1}(\cdot)-2 W_{\mathrm{cl}}(\mathrm{Id})$ which is independent from $\rho$ and $y$. The trial function $y(x)=x$ yields the upper bound $\sigma_{\text {end }}^{(\rho)} \leq-V_{1}(1)-2 V_{2}(2)$. Now we use the uniform convexity of $V_{1}$ and $V_{2}$ in order to conclude that $y^{(\rho)}$ satisfies the a priori bound

$$
\sum_{k=0}^{\rho-1}\left|y^{(\rho)}(x+1)-y^{(\rho)}(x)-1\right|^{2} \leq C
$$

for some constant $C$ that does not depend on $\rho$.

Assume now that $\left(\rho_{k}\right)_{k \in \mathbb{N}}$ is a subsequence such that $\lim _{k \rightarrow \infty} \sigma_{\text {end }}^{\left(\rho_{k}\right)}=\liminf _{\rho \rightarrow \infty} \sigma_{\text {end }}^{(\rho)}=\sigma$. Let $y_{k}=y^{\left(\rho_{k}\right)}$, choose a pair of numbers $\delta>0, k \in \mathbb{N}$ such that $\left|\sigma^{\left(\rho_{k}\right)}-\sigma\right|<\delta / 2$, let $C_{L}$ be the Lipschitz constant of the pair energy (1.11) within the ball given by the a priori bound (6.2). Increasing $k$ further if necessary we find $x_{0}$ such that $\max \left\{\left|y_{k}\left(x_{0}\right)-y_{k}\left(x_{0}-1\right)-1\right|,\left|y_{k}\left(\rho_{k}-x_{0}+1\right)-y_{k}\left(\rho_{k}-x_{0}\right)-1\right|\right\}<\delta /\left(4 C_{L}\right)$. For arbitrary $\rho$ we define now

$$
y_{\text {trial }}^{(\rho)}(x)=\left\{\begin{aligned}
y_{k}(x)-y_{k}\left(x_{0}\right)+x_{0} & \text { if } x \in\left[0, x_{0}\right] \cap \mathbb{Z}, \\
y_{k}\left(x-\rho+\rho_{k}\right)-y_{k}\left(\rho_{k}-x_{0}\right)+\rho-x_{0} & \text { if } x \in\left[\rho-x_{0}, \rho\right] \cap \mathbb{Z}, \\
x & \text { else. }
\end{aligned}\right.
$$

The trial function $y_{\text {trial }}^{(\rho)}$ gives the upper bound $\sigma_{\text {end }}^{(\rho)} \leq \sigma_{\text {end }}^{\left(\rho_{k}\right)}+\delta / 2 \leq \sigma+\delta$. Since $\delta$ is arbitrarily small this proves claim A).

B) We assume without loss of generality that $V_{1}(1)=V_{2}(2)=0$ and define $\eta^{(\rho)}(x)=y^{(\rho)}(x+1)-y^{(\rho)}(x)-1$. The uniform convexity together with (1.10) implies that $V_{i}(\cdot) \geq-C \varepsilon^{2}$ for some constant $C>0$. Use the the lower bound (6.1) to derive the improved a priori estimate

$$
\sum_{x=0}^{\rho-1}\left|\eta^{(\rho)}(x)\right|^{2} \leq C \varepsilon^{2}
$$


The differentiability of $V_{1}$ and $V_{2}$ implies that

$$
\begin{aligned}
\sigma_{\text {end }}^{(\rho)}= & \sum_{x=0}^{\rho-1} V_{1}\left(1+\eta^{(\rho)}(x)\right)+\sum_{x=0}^{\rho-2} V_{2}\left(2+\eta^{(\rho)}(x)+\eta^{(\rho)}(x+1)\right) \\
= & \sum_{x=0}^{\rho-1}\left[V_{1}^{\prime}(1) \eta^{(\rho)}(x)+\frac{1}{2} V_{1}^{\prime \prime}(1)\left(\eta^{(\rho)}(x)\right)^{2}\right] \\
& +\sum_{x=0}^{\rho-1}\left[V_{2}^{\prime}(2)\left(\eta^{(\rho)}(x)+\eta^{(\rho)}(x+1)\right)+\frac{1}{2} V_{2}^{\prime \prime}(2)\left(\eta^{(\rho)}(x)+\eta^{(\rho)}(x+1)\right)^{2}\right] \\
& +\sum_{x=0}^{\rho-1} w_{1}\left(\eta^{(\rho)}(x)\right)+\sum_{x=0}^{\rho-2} w_{2}\left(\eta^{(\rho)}(x)+\eta^{(\rho)}(x+1)\right),
\end{aligned}
$$

where $\lim _{\eta \rightarrow 0} \eta^{-2} w_{1}(\eta)=\lim _{\eta \rightarrow 0} \eta^{-2} w_{2}(\eta)=0$. The assumptions on $V_{1}$ and $V_{2}$ entail that $V_{1}^{\prime}(1)=\frac{1}{2} \varepsilon$, $V_{2}^{\prime}(2)=-\frac{1}{4} \varepsilon$. We apply the bound (6.3) and formula (6.4), set $z=\frac{1}{\varepsilon} \eta$ and obtain that

$$
\lim _{\rho \rightarrow \infty} \frac{1}{\varepsilon^{2}} \sigma=+\frac{1}{4}(z(0)+z(\rho-1))+\sum_{x=0}^{\rho-1} \frac{K_{1}}{2}(z(x))^{2}+\sum_{x=0}^{\rho-2} \frac{K_{2}}{2}(z(x)+z(x+1))^{2} .
$$

We will now compute the ground state $z_{\min }$ of the right-hand side. The equilibrium condition of (6.5) is given by

$$
\begin{gathered}
K_{1} z(x)+K_{2}(z(x+1)+2 z(x)+z(x-1))=0, \text { if } x>0 \\
K_{1} z(0)+K_{2}(z(0)+z(1))=\frac{1}{4} .
\end{gathered}
$$

We plug the Ansatz $z(x)=z_{0} \lambda^{x}$ into the equilibrium condition and solve the characteristic equation $K_{1} \lambda+$ $K_{2}\left(\lambda^{2}+2 \lambda+1\right)=0$ for $\lambda$ within the set $|\lambda|<1$ :

$$
\lambda=\frac{1}{2}\left(\sqrt{4 \frac{K_{1}}{K_{2}}+\frac{K_{1}^{2}}{K_{2}^{2}}}-\frac{K_{1}}{K_{2}}-2\right) .
$$

Now we solve the second equation for $z_{0}$ and obtain that

$$
z_{0}=-\frac{1}{4} \frac{1}{K_{1}+(1+\lambda) K_{2}}=-\frac{1}{2} \frac{1}{\sqrt{4 K_{1} K_{2}+K_{1}^{2}}+K_{1}} .
$$

The energy of the Ansatz $z(x)=z_{0} \lambda^{x}$ is given by

$$
-\frac{1}{4} z_{0}+\frac{z_{0}}{2} \frac{K_{1}+(1+\lambda)^{2} K_{2}}{1-\lambda^{2}}
$$

replace $z_{0}$ and $\lambda$ by the previously derived expressions to obtain the formula

$$
\lim _{\rho \rightarrow \infty} \frac{1}{\varepsilon^{2}} \sigma=\frac{1}{64 K_{2}}\left(\sqrt{1+4 K_{2} / K_{1}}-1\right) .
$$


It can be checked without difficulty that the trial function

$$
y_{\text {trial }}(x)=x+\varepsilon\left\{\begin{array}{cc}
-\sum_{k=x}^{\infty} z_{\min }(k), & \text { if } x \in[0, \rho / 2] \cap \mathbb{Z}, \\
\sum_{k=x+1}^{\rho} z_{\min }(k), & \text { if } x \in(\rho / 2, \rho] \cap \mathbb{Z}
\end{array}\right.
$$

realizes the ground state energy of the right-hand side in (6.5) as $\rho \rightarrow \infty$.

\section{REFERENCES}

[1] R. Alicandro, A. Braides and M. Cicalese, Continuum limits of discrete films with superlinear growth densities. Calc. Var. Par. Diff. Eq. 33 (2008) 267-297.

[2] S. Aubry, The twist map, the extended Frenkel-Kontorova model and the devil's staircase. Physica D 7 (1983) $240-258$.

[3] X. Blanc, C. Le Bris and P.L. Lions, From molecular models to continuum mechanics. Arch. Rat. Mech. Anal. 164 (2002) $341-381$.

[4] A. Braides and M. Cicalese, Surface energies in nonconvex discrete systems. Math. Models Meth. Appl. Sci. 17 (2007) 985-1037.

[5] A. Braides and A. DeFranchesi, Homogenisation of multiple integrals. Oxford University Press (1998).

[6] A. Braides and M. Gelli, Continuum limits of discrete systems without convexity hypotheses. Math. Mech. Solids 7 (2002) 41-66.

[7] A. Braides, M. Solci and E. Vitali, A derivation of linear alastic energies from pair-interaction atomistic systems. Netw. Heterog. Media 9 (2007) 551-567.

[8] J. Cahn, J. Mallet-Paret and E. Van Vleck, Travelling wave solutions for systems of ODEs on a two-dimensional spatial lattice. SIAM J. Appl. Math. 59 (1998) 455-493.

[9] M. Charlotte and L. Truskinovsky, Linear elastic chain with a hyper-pre-stress. J. Mech. Phys. Solids 50 (2002) $217-251$.

[10] W. E and P. Ming, Cauchy-Born rule and the stability of crystalline solids: static problems. Arch. Rat. Mech. Anal. $\mathbf{1 8 3}$ (2005) 241-297.

[11] I. Fonseca and S. Müller, A uniqueness proof for the Wulff theorem. Proc. Roy. Soc. Edinburgh Sect. A 119 (1991) $125-136$.

[12] G. Friesecke and F. Theil, Validitity and failure of the Cauchy-Born rule in a two-dimensional mass-spring lattice. J. Nonlinear Sci. 12 (2002) 445-478.

[13] G. Friesecke, R. James and S. Müller, A theorem on geometric rigidity and the derivation of nonlinear plate theory from three-dimensional elasticity. Comm. Pure Appl. Math. 55 (2002) 1461-1506.

[14] D. Gérard-Varet and N. Masmoudi, Homogenization and boundary layer. Preprint available at www.math.nyu.edu/faculty/ masmoudi/homog_Varet3.pdf (2010).

[15] P. Lancaster and L. Rodman, Algebraic Riccati Equations. Oxford University Press (1995).

[16] J.L. Lions, Some methods in the mathematical analysis of systems and their controls. Science Press, Beijing, Gordon and Breach, New York (1981).

[17] J.A. Nitsche, On Korn's second inequality. RAIRO Anal. Numér. 15 (1981) 237-248.

[18] C. Radin, The ground state for soft disks. J. Stat. Phys. 26 (1981) 367-372.

[19] B. Schmidt, A derivation of continuum nonlinear plate theory from atomistic models. Multiscale Mod. Sim. 5 (2006) 664-694.

[20] B. Schmidt, On the passage from atomic to continuum theory for thin films. Arch. Rat. Mech. Anal. 190 (2008) 1-55.

[21] B. Schmidt, On the derivation of linear elasticity from atomistic models. Net. Heterog. Media 4 (2009) $789-812$.

[22] E. Sonntag, Mathematical Control Theory. Second edition, Springer (1998).

[23] L. Tartar, The general theory of homogenization. Springer (2010).

[24] F. Theil, A proof of crystallization in a two dimensions. Comm. Math. Phys. 262 (2006) $209-236$. 